\title{
Inherited thrombocytopenias: history, advances and perspectives
}

\author{
Alan T. Nurden and Paquita Nurden \\ Institut Hospitalo-Universitaire LIRYC, Pessac, France
}

Haematologica 2020

Volume 105(8):2004-2019

\section{Correspondence: \\ ALAN NURDEN \\ nurdenat@gmail.com \\ Received: April 23, 2020. \\ Accepted: May 8, 2020. \\ Pre-published: June 11, 2020.}

doi:10.3324/haematol.2019.233197

Check the online version for the most updated information on this article, online supplements, and information on authorship \& disclosures: www.haematologica.org/content/105/8/2004

(C)2020 Ferrata Storti Foundation

Material published in Haematologica is covered by copyright. All rights are reserved to the Ferrata Storti Foundation. Use of published material is allowed under the following terms and conditions:

https://creativecommons.org/licenses/by-nc/4.0/legalcode. Copies of published material are allowed for personal or internal use. Sharing published material for non-commercial purposes is subject to the following conditions:

https://creativecommons.org/licenses/by-nc/4.0/legalcode, sect. 3. Reproducing and sharing published material for commercial purposes is not allowed without permission in writing from the publisher.

\section{ABSTRACT}

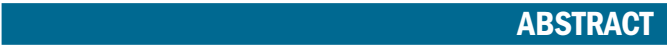

$\mathrm{O}$ ver the last 100 years the role of platelets in hemostatic events and their production by megakaryocytes have gradually been defined. Progressively, thrombocytopenia was recognized as a cause of bleeding, first through an acquired immune disorder; then, since 1948, when Bernard-Soulier syndrome was first described, inherited thrombocytopenia became a fascinating example of Mendelian disease. The platelet count is often severely decreased and platelet size variable; associated platelet function defects frequently aggravate bleeding. Macrothrombocytopenia with variable proportions of enlarged platelets is common. The number of circulating platelets will depend on platelet production, consumption and lifespan. The bulk of macrothrombocytopenias arise from defects in megakaryopoiesis with causal variants in transcription factor genes giving rise to altered stem cell differentiation and changes in early megakaryocyte development and maturation. Genes encoding surface receptors, cytoskeletal and signaling proteins also feature prominently and Sanger sequencing associated with careful phenotyping has allowed their early classification. It quickly became apparent that many inherited thrombocytopenias are syndromic while others are linked to an increased risk of hematologic malignancies. In the last decade, the application of next-generation sequencing, including whole exome sequencing, and the use of gene platforms for rapid testing have greatly accelerated the discovery of causal genes and extended the list of variants in more common disorders. Genes linked to an increased platelet turnover and apoptosis have also been identified. The current challenges are now to use next-generation sequencing in first-step screening and to define bleeding risk and treatment better.

\section{History}

In the late $19^{\text {th }}$ century improvements to the light microscope led to anucleate platelets being visualized in great numbers in human blood. Early pioneers in the field of platelet research included the Canadian William Osler, a Paris hematologist, George Hayem, who performed the first accurate platelet count, and the Italian Giulio Bizzozero. ${ }^{1}$ In 1906, James Homer Wright confirmed that platelets were produced by bone marrow megakaryocytes. ${ }^{2}$ When, in 1951, Harrington et al. ${ }^{3}$ observed purpura in a child of a mother with immune thrombocytopenic purpura, a maternal factor was said to be destroying the platelets. Anti-platelet antibodies were identified as the cause and platelet transfusions, immunosuppressive therapy and splenectomy became standard treatments. Acquired thrombocytopenia, often with defective platelet function, has many causes. For example, it may be an immune response linked to blood transfusions and drugs, a direct consequence of viral or bacterial infections, be the result of other hematologic disorders or be secondary to many major illnesses. However, some thrombocytopenias are inherited and Professors Jean Bernard and Jean-Pierre Soulier in Paris were pioneers in this domain, describing in 1948 what they called in French "dystrophie thrombocytaire hémorragipare congénitale", later re-named the Bernard-Soulier syndrome (BSS). ${ }^{4}$ Strikingly, many platelets were enlarged and some were giant. A key to the molecular defect was the platelet deficit of sialic acid, a negatively charged monosaccharide terminating many of the oligosaccharides of platelet glycoproteins (GP) and 
some glycolipids. ${ }^{5}$ The discovery that a major component of the platelet surface, first identified as "GPI", was absent from platelets in BSS began the long road to defining the molecular landscape of inherited thrombocytopenias. ${ }^{6}$ This centenary review will take a concise look at this journey emphasizing major steps in the current understanding of these conditions and highlighting recent advances in what is an intriguing subset of Mendelian diseases.

\section{Platelet production and lifespan}

The platelet count is the result of a balance between the biogenesis, senescence and consumption of these cells. Briefly, CD34+ hematopoietic stem cells (HSC) first give rise to megakaryocyte-erythrocyte progenitors, a proportion of which will develop into colonies that mature by endomitosis to polylobulated megakaryocytes with a high DNA content (Figure 1). Megakaryocyte differentiation is regulated by transcription factors as well as by interactions with chemokines, cytokines and constituents of the extracellular matrix. ${ }^{7}$ Thrombopoietin, acting through its receptor $\mathrm{Mpl}$, is a key factor. ${ }^{8}$ Mostly produced in the liver, thrombopoietin levels in blood are governed by the platelet number; feedback mechanisms stimulate HSC proliferation and megakaryopoiesis. The demarcation membrane system of mature megakaryocytes is a membrane reservoir for platelet biogenesis. Migrating to the endothelial cell sinusoid barrier, mature megakaryocytes extend long, branched, protrusions, termed proplatelets, into the blood where under flow they release large numbers of platelets from their ends. ${ }^{9,10}$ This whole process is highly dependent on cytoskeletal proteins including myosin IIA, actin filaments and the tubulins that provide tracks for the transport of organelles with a Cdc42/RhoA regulatory circuit guiding transendothelial platelet biogenesis. ${ }^{11}$ Type I collagen in the extracellular matrix is a negative regulator, preventing premature proplatelet formation. Early reports also insisted that megakaryocytes can migrate directly into the blood and accumulate in the lungs. Using two-photon microscopy, in vivo lineage tracing technologies and a series of lung transplants in mouse models, Lefrancais et al. ${ }^{12}$ have confirmed that the lung is indeed a site for platelet biogenesis. In conditions of acute need, direct megakaryocyte rupture is an alternative pathway for platelet production. ${ }^{13}$

If unused in hemostatic events or pathological processes, human platelets circulate for 7-10 days (Figure 2). Platelet clearance due to senescence occurs in the liver. Loss of sialic acid through the action of neuraminidase during platelet aging exposes galactose or $\mathrm{N}$-acetyl-galactosamine, residues that mediate platelet binding to the

\section{Selected Genes: Impaired Megakaryopoiesis and Platelet Biogenesis}

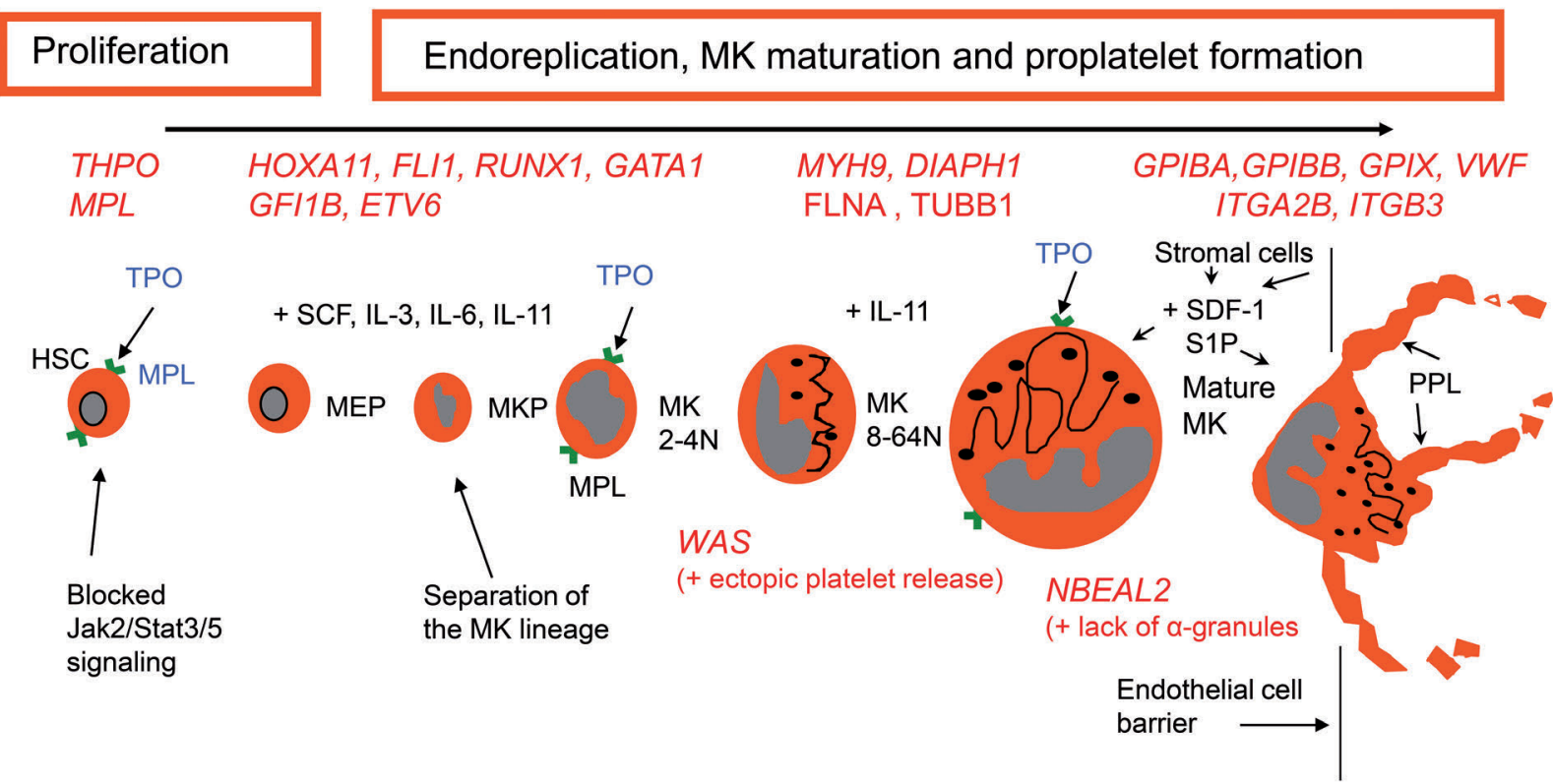

Bone marrow

Vascular sinus

Figure 1. A schema of the major steps of megakaryopoiesis highlighting how inherited defects of selected genes cause thrombocytopenia. Megakaryocytes arise from hematopoietic stem cells that proliferate to first form megakaryocyte-erythrocyte progenitors, a proportion of which give rise to colony-forming megakaryocyte progenitors. These events require the interaction of thrombopoietin with its receptor and are under the influence of various growth factors (e.g. stem cell factor, cytokines and interleukins). The developing megakaryocytes undergo endomitosis to increase chromosome number; they then mature before migrating to the vascular sinus where they extend proplatelets or migrate themselves across the endothelial cell barrier into the vascular sinus. This latter step is under the influence of many factors including stromal derived growth factor-1 and sphingosine-1-phosphate. Selected genes with variants causing inherited thrombocytopenias are shown in red. HSC: hematopoietic stem cells; TPO: thrombopoietin; MPL: thrombopoietin receptor; MEP: megakaryocyte-erythrocyte progenitor; SCF: stem cell factor; IL: interleukin; MKP: megakaryocyte progenitor, MK: megakaryocyte; SDF-1: stromal derived growth factor-1; S1P: sphingosine-1-phosphate; PPL: proplatelet. 
hepatocyte Ashwell-Morell receptor, an act that stimulates the synthesis of thrombopoietin. ${ }^{14}$ Subsequent studies using a mouse model additionally pointed to clearance by Kupffer cells in the liver sinusoids; here $\mathrm{N}$-acetyl-galactosamine was recognized by the lectin, CLEC4A. ${ }^{15}$ Stateof-the-art microscopy then pinpointed how Küpffer cells also recognized $\beta$-galactose on aged mouse or human platelets with a galactose-binding lectin mediating the phagocytosis. ${ }^{16}$ Most importantly, Küpffer cells were shown to continually scan circulating platelets with touchand-go interactions as these latter pass through sinusoidal endothelial fenestrae.

In megakaryocytes, the role of apoptosis during proplatelet formation and platelet biogenesis is controversial and largely unproven although it may intervene after platelet release or during stress situations such as infections and inflammation. ${ }^{17}$ In a classic paper, the pro-survival protein, $\mathrm{Bcl}-\mathrm{X}_{\mathrm{L}}$, was proposed to constrain the prodeath Bak in platelets; the balance acting as a molecular clock for platelet survival. ${ }^{18}$ Others have shown how apoptotic pathways and necrosis engage pro-death proteins triggering mitochondrial membrane permeabilization, cytochrome $\mathrm{C}$ release and caspase activation leading to $\mathrm{Ca}^{2+}$-dependent exposure of phosphatidylserine, which is not only recognized by phagocytic cells but also leads to procoagulant activity. ${ }^{19}$

The following sections of this review will describe familial defects of platelet production and megakaryopoiesis; affected genes will be shown to interfere with HSC proliferation, megakaryocyte maturation and migration and the alteration of platelet lifespan. Many are accompanied by an increased platelet size (Figure 2) while others have a normal platelet size or even small platelets (Figure 3). Platelet function is variably affected. While some thrombocytopenias are isolated, many are syndromic and/or associated with other conditions that may be of major clinical importance. Bleeding is mostly mucocutaneous, severe in some disorders, but infrequent or even absent in others, particularly when the decrease in platelet count is modest. A summary of the genes involved and the principle characteristics of each disorder are presented in Online Supplementary Table S1.

\section{Classic inherited thrombocytopenias}

We begin with a series of named inherited thrombocytopenias whose characterization has marked the history of inherited platelet disorders. In many of these syndromes, thrombocytopenia, defined as a platelet count $<150 \times 10^{9} / \mathrm{L}$, is accompanied by platelet function defects that aggravate bleeding. In early studies genotyping mostly involved candidate gene sequencing and linkage studies.

\section{Bernard-Soulier syndrome}

The "GPI" lesion in BSS platelets was quickly shown to involve a complex of four distinct subunits each encoded by a distinct gene. The large, heavily glycosylated GPIb $\alpha$ is attached by a disulfide to the small GPIb $\beta$, while GPIX and GPV are non-covalently associated, all in a 2:2:2:1 stoichiometry. Bleeding in BSS patients is disproportionate to

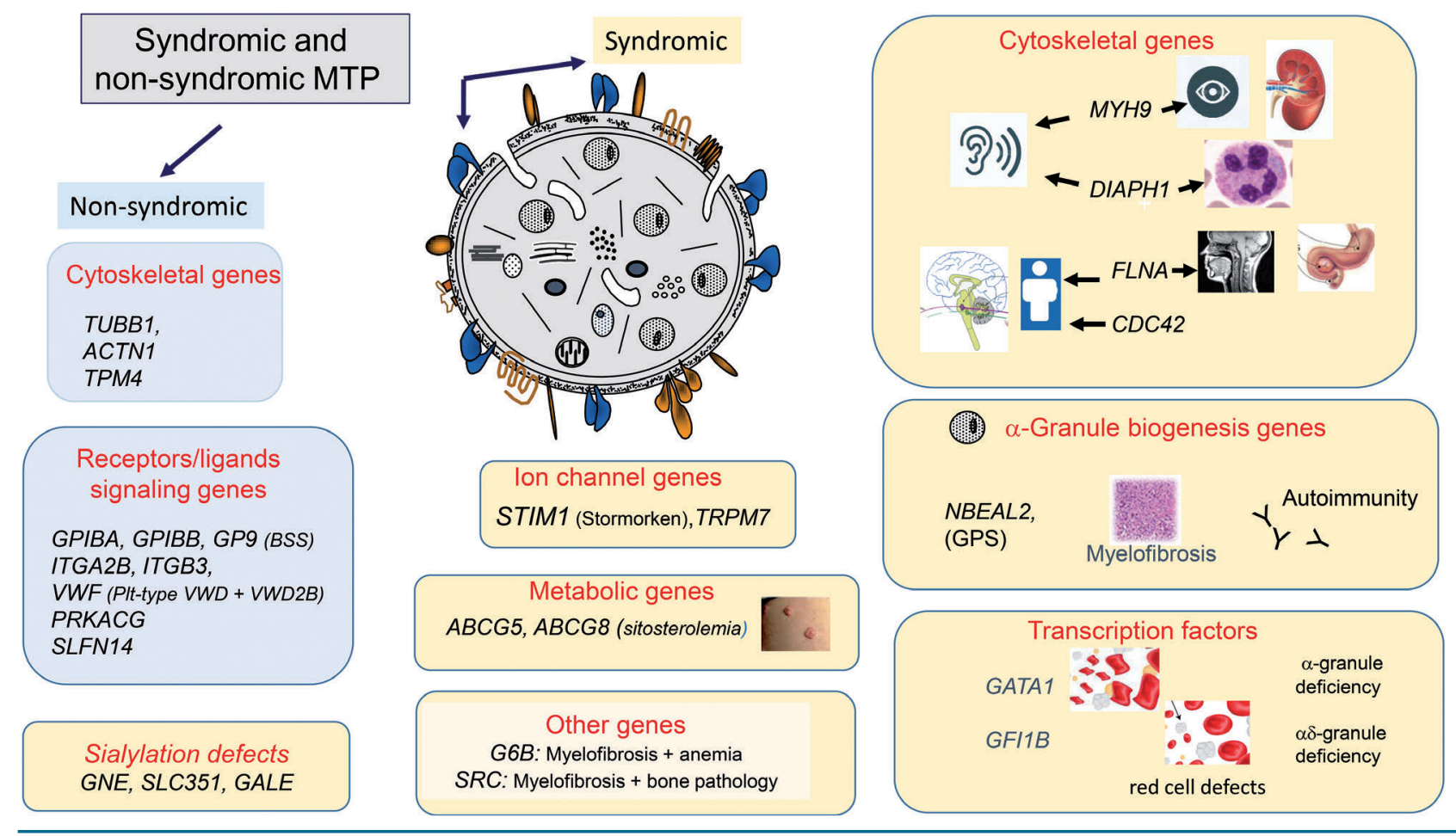

Figure 2. A cartoon showing genes causing non-syndromic and syndromic macrothrombocytopenias. The causative genes are grouped according to the nature of the encoded protein and/or the secondary condition(s) that may accompany the macrothrombocytopenia. MTP: macrothrombocytopenias; BSS: Bernard-Soulier syndrome; pl-type VWD: platelet-type von Willebrand syndrome; VWD2B: von Willebrand disease type 2B; GPS: gray platelet syndrome. 
the degree of thrombocytopenia because the absence of GPIb $\alpha$ prevents the shear-dependent tethering of platelets to von Willebrand factor (VWF) in vascular lesions, an abnormality mimicked by the loss of ristocetin-induced platelet agglutination in a diagnostic test. ${ }^{20,21}$ The search for mutations first targeted GP1BA, the gene encoding GPIb $\alpha$, but mutations in $G P 1 B B$ and GP9 were also quickly shown to cause BSS by preventing the surface expression of GPIb ${ }^{22}$ The generation and rescue of BSS in a mouse model confirmed the link between GPIb $\alpha$ loss and the appearance of giant platelets and, therefore, macrothrombocytopenia. ${ }^{23}$ Typical findings in BSS and the mouse models are aberrant formation of the demarcation membrane system in megakaryocytes while fewer proplatelets protrude into the vascular sinus and these proplatelets are thicker with larger heads. While the loss of megakaryocyte interactions with extracellular proteins remains a plausible molecular basis of BSS, the absence of mechanical stabilizing interactions between GPIb, cytoskeletal proteins and internal membranes is another likely factor.

While classic BSS has autosomal recessive (AR) inheritance, mono-allelic forms with autosomal dominant (AD) transmission are a frequent cause of mild macrothrombocytopenia in Europe. The initial example was the Bolzano (p.A172V) mutation affecting GPIbo in Italian families, said to be responsible for Mediterranean macrothrombocytopenia, although other variants of GP1BA have since been described. More recently a series of single allele variants of GPIBB have been identified by whole exome sequencing (WES) in patients with mild macrothrombocytopenia. ${ }^{24}$ The difference in phenotype given by $\mathrm{AD}$ single allelic forms of BSS compared with heterozygosity for biallelic BSS has yet to be fully explained. A 1.5 to $3.0-\mathrm{Mb}$ hemizygous mostly somatic deletion on chromosome 22 q11.2 including GPIBB is seen in the Di-George and velocardiofacial syndromes in which multiple developmental defects are often accompanied by mild to moderate macrothrombocytopenia. ${ }^{21}$

\section{Platelet-type von Willebrand disease and type 2B von Willebrand disease}

GPIb $\alpha$ has seven leucine-rich repeats and flanking regions near its $\mathrm{N}$-terminus; the mucin-like domain follows with the many negatively charged $O$-linked oligosaccharides that provide rigidity. In platelet-type von Willebrand disease, $\mathrm{AD}$ gain-of-function missense mutations within the leucine-rich repeats (and 1 deletion outside the repeats) promote spontaneous binding of large VWF multimers. ${ }^{25}$ As a result, the higher molecular weight multimers are decreased or absent in plasma. Cross-linking of platelets by VWF favors platelet clumping and a high sensitivity to ristocetin-induced platelet agglutination. In culture, spontaneous binding of VWF multimers to maturing megakaryocytes inappropriately activates intracellular signaling pathways; as a result, there are fewer proplatelets and they have enlarged tips. ${ }^{26}$ Furthermore, VWF-bound platelets are rapidly removed from the circulation in a process that is enhanced when aggregates are present. Bleeding is accentuated under conditions of stress, such as pregnancy when circulating large VWF multimers are elevated. Mention should also be made of type $2 \mathrm{~B}$ von Willebrand disease, also with $\mathrm{AD}$ inheritance. In this condition single allele

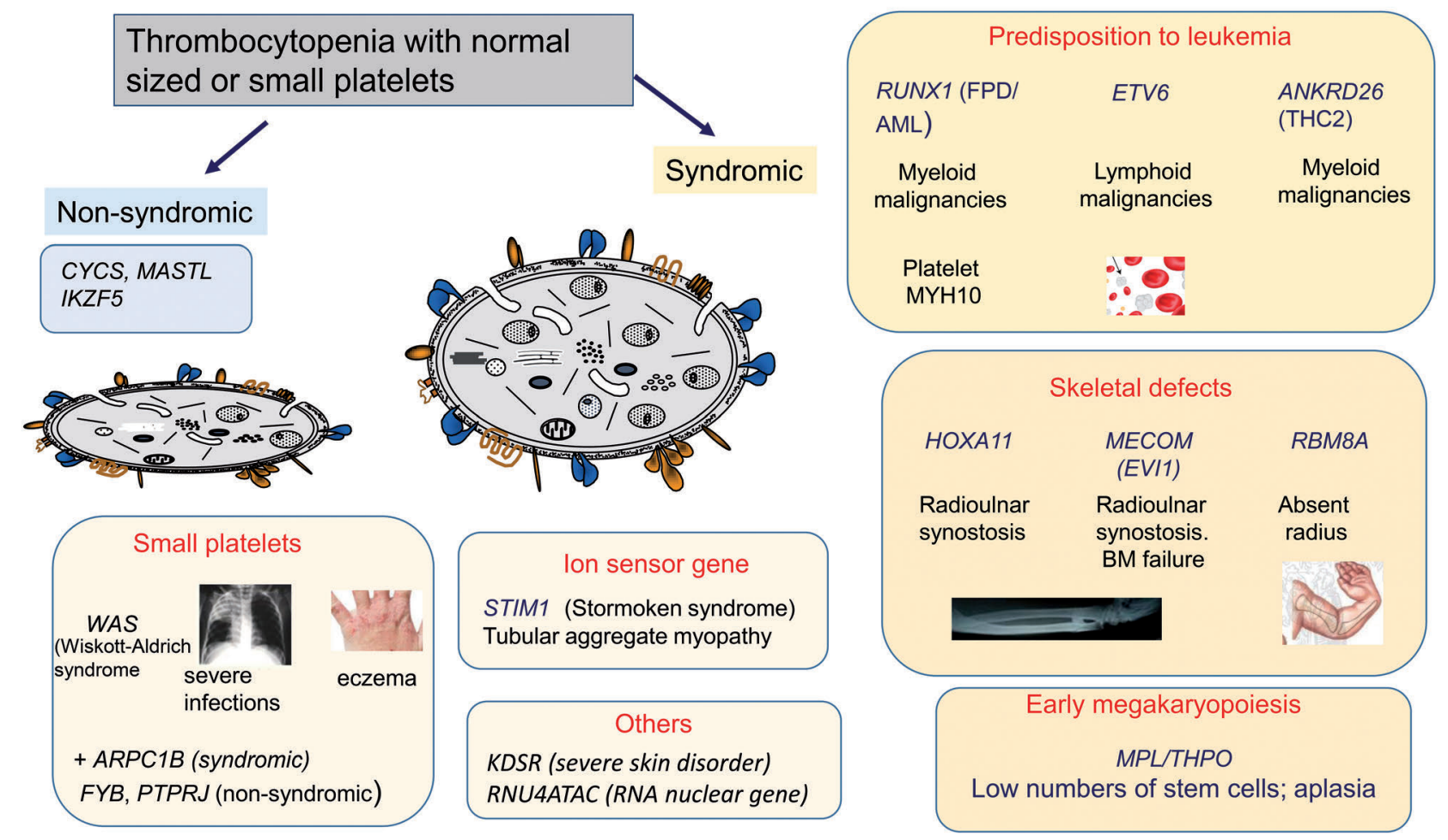

Figure 3. A cartoon showing genes causing non-syndromic and syndromic thrombocytopenias with normal sized or small platelets. The genes are grouped according to the nature of the encoded abnormal protein and to the accompanying secondary condition(s) that define the syndrome. BM: bone marrow. 
gain-of-function mutations within exon 28 of VWF result in multimers that bind spontaneously to GIb $\alpha .^{27}$ Enlarged platelets and thrombocytopenia are features and the crosslinking of platelets by adsorbed VWF multimers leads to platelet clumping in many patients. Megakaryocytes in culture appear to bind VWF multimers leaking from their own cells; the mature megakaryocytes have larger platelet territories while proplatelet-like protrusions are fewer, shorter and the swellings larger. ${ }^{28}$ Patients of French-Canadian descent with what was called the "Montreal platelet syndrome", an inherited macrothrombocytopenia, in fact possessed the VWF p.V1316M mutation. Recent studies on both a mouse model and platelets from a patient with VWF $\mathrm{V} 1316 \mathrm{M}$ have shown that platelet aggregation is also reduced due to the loss of essential signaling requirements for $\alpha I l b \beta 3$ activation. ${ }^{29}$ With regards to a possible loss of sialic acid from platelets of these patients, the same group showed that while endogenous neuraminidase translocated to the platelet surface, the desialylation threshold required for thrombocytopenia in vivo was not reached; intriguingly, $\mathrm{N}$-glycans on $\alpha I I b$ and $\beta 3$ and not GPIb $\alpha$, were most targeted. ${ }^{30}$ But both platelet-type von Willebrand disease and type $2 \mathrm{~B}$ von Willebrand disease are disorders in which increased platelet turnover contributes to the thrombocytopenia.

\section{MYH9-related disease}

Myosin, heavy chain 9 (MYH9), a subunit of myosin IIA, mediates intracellular contractile forces generated through ATP hydrolysis and the actin cytoskeleton. ADinherited $M Y H 9$ variants are a common cause of moderate to severe macrothrombocytopenia and platelets can be very large. ${ }^{31}$ Cytochemical or immunofluorescence detection of inclusions (Döhle-like bodies) in leukocytes on blood smears facilitates the diagnosis. ${ }^{32}$ Myosin IIA is expressed in many tissues and MYH9-related disease will often become syndromic with age; symptoms include glomerulonephritis, potentially leading to renal failure (requiring dialysis or transplantation), sensorial hearing loss, early cataracts and elevated circulating liver enzymes indicating hepatic damage. Historically, combinations of macrothrombocytopenia with these conditions were known as May-Hegglin anomaly, and Epstein, Fechtner and Sebastian syndromes but all were later linked to mutations in $M Y H 9{ }^{33}$ Disease-causing variants are mostly missense but variants of all types have been described and affect both the N-terminal head or motor domain and Cterminal tail (responsible for dimerization and containing phosphorylation sites). ${ }^{33,34}$ Some residues are mutational hotspots, for example S96 and R702 in the head and R1165 and D1424 in the tail and genotype-phenotype correlations have been linked to disease evolution in MYH9related disease. Notwithstanding the $\mathrm{AD}$ inheritance, some of the mutations are sporadic and somatic germinal mosaicism has been reported. Lacking contractile force, affected megakaryocytes show reduced migration to the vascular sinus and, together with altered proplatelet formation, this results in ectopic but reduced platelet release in the marrow. ${ }^{35}$ Treatment includes slowing the extrahematologic effects with cochlear or kidney transplantation and renin-angiotensin inhibitors in some cases.

\section{Gray platelet syndrome and related disorders.}

A qualitative disorder characterized by moderate macrothrombocytopenia with "gray-colored" platelets on a stained blood smear, gray platelet syndrome has mostly
AR inheritance. Patients have enlarged platelets lacking $\alpha$ granules and their storage pool of proteins. ${ }^{36}$ Thrombocytopenia is often progressive and bleeding highly variable. Enlarged spleens, myelofibrosis, high serum vitamin B12 levels and reduced platelet function help define the phenotype. ${ }^{37}$ In 2011 , three groups using different approaches including next-generation WES and RNA profiling showed that mutations in NBEAL2 caused gray platelet syndrome in large, but distinct cohorts of patients (data reviewed by Chen et al. ${ }^{38}$ ). NBEAL2 is a scaffolding protein involved in $\alpha$-granule ontogeny. Mouse Nbeal2 models recapitulated the gray platelet syndrome phenotype and showed how the lack of the secretory pool of biologically active proteins affects wound healing, and has consequences for thrombosis and inflammation. ${ }^{39}$ Cultured megakaryocytes from patients with NBEAL2 mutations interacted abnormally with extracellular matrix proteins, including type I collagen, with reduced proplatelet formation and branching. ${ }^{40}$ Extensive emperipolesis of neutrophils by the megakaryocytes is another feature of gray platelet syndrome. Although altered neutrophil structure and increased infections were reported in early studies on this syndrome, only recently was a role for NBEAL2 in immunity confirmed. ${ }^{41} \mathrm{New}$ avenues for research on autoimmunity and inflammation in gray platelet syndrome have now opened.

The absence of platelet $\alpha$-granules is also a characteristic of children with arthrogryposis, renal dysfunction and cholestasis (ARC) syndrome, a severe AR multisystem disorder especially affecting the kidneys and linked to mutations in VPS33B and VPS16B, genes encoding proteins involved in $\alpha$-granule biogenesis; however, platelet count and size are often normal and this syndrome is not included in Online Supplementary Table $S 1{ }^{38}$ A paucity of $\alpha$-granules in platelets of patients with X-linked mutations in GATA1 is explained by its long-distance regulation of NBEAL2. ${ }^{42}$ The transcription factor, GATA1, in complex with its cofactor FOG1 acts as a gene repressor in hematopoietic cell lineages including megakaryocytes and red blood cells. It was first identified as a cause of macrothrombocytopenia with moderate to severe bleeding by Nichols et al., who found a p.V205M mutation in siblings with severe thrombocytopenia and dyserythropoietic anemia. ${ }^{43}$ Most GATA1 mutations result in dysmegakaryopoiesis with the marrow often containing an abundance of small megakaryocytes; the red blood cell abnormalities include features that can be observed in $\beta$ thalassemia and also congenital erythropoietic porphyria. ${ }^{21}$ Phenotypic variation is considerable and the condition often improves with age. A loss of collagen-induced platelet aggregation in some patients remains unexplained. Macrothrombocytopenia, an absence of platelet $\alpha$-granules and red blood cell defects variably characterize patients with $\mathrm{AD}$ germline mutations in $\mathrm{GFI} 1 \mathrm{~B}$, a transcriptional repressor and key regulator of hematopoiesis. ${ }^{44,45}$ The macrothrombocytopenia can be accompanied by platelet function defects and myelofibrosis; bleeding is mostly mild or even absent but may be severe after trauma. Abnormal megakaryocyte maturation and proplatelet formation contribute to the macrothrombocytopenia. The phenotype depends on the site and nature of the mutation and the GFIAB isoform affected. ${ }^{46}$ Haploinsufficiency or non-functioning of GATA1 or GFI1B will change the activation of a number of genes coding for proteins maintaining platelet function. 


\section{Congenital amegakaryocytic thrombocytopenia}

Born with severe thrombocytopenia but normal sized platelets, patients with congenital amegakaryocytic thrombocytopenia lack mature megakaryocytes in the bone marrow. Evolution to critical multi-lineage aplasia occurs before adulthood..$^{4748}$ In the majority of cases, AR mutations in the MPL gene make the cells incapable of binding thrombopoietin; as a result circulating thrombopoietin levels are very high. ${ }^{47}$ Nonsense mutations with a complete loss of Mpl give rise to a severe form (type I) while missense mutations and the presence of residual $\mathrm{Mpl} \mathrm{may} \mathrm{give} \mathrm{a} \mathrm{milder} \mathrm{form} \mathrm{of} \mathrm{the} \mathrm{disease} \mathrm{(type} \mathrm{II).} \mathrm{The}$ mutations lead to a loss of thrombopoietin binding or on rare occasions, altered receptor recycling. In culture, megakaryocytes from the patients with congenital amegakaryocytic thrombocytopenia fail to form colonies. The mutations can also affect stem cell maintenance. Disease severity depends on the extent of the interference with JAK2/STAT and MAPK signaling pathways, with there being residual signaling in type II disease. HSC transplantation has been the treatment of choice. ${ }^{48}$ Progression to aplastic anemia or myelodysplastic syndrome is a risk. Rare homozygous mutations in the THPO gene with loss of thrombopoietin production or an inability to bind to $\mathrm{Mpl}$ can also lead to bone marrow aplasia. Note that because of the largely hepatic origin of thrombopoietin, these patients are unsuitable for bone marrow transplantation. ${ }^{48}$ Conversely, both MPL and THPO gain-of-function mutations are a source of familial thrombocytosis.

\section{Wiskott-Aldrich syndrome}

Wiskott-Aldrich syndrome (WAS) is an X-linked disorder described by Alfred Wiskott in 1937 and Robert Aldrich in 1954. The syndrome is characterized by severe immunodeficiency, thrombocytopenia and small platelets. The clinical spectrum includes eczema, infections, autoimmune hemolytic anemia and cancer; bleeding is mostly mild but can be severe if the platelet count is very low and can prove fatal. ${ }^{49}$ Hemizygous mutations in the WAS gene abrogate expression or alter function of WAS protein: the protein has multiple roles in the early stages of actin polymerization and in signal transduction with virtually all hematopoietic lineages affected. ${ }^{50,51}$ Megakaryocytes of WAS patients form proplatelets prematurely and shed platelets ectopically in the marrow. ${ }^{52}$ There is also a peripheral component to the thrombocytopenia with circulating autoantibodies and platelet destruction in the spleen making WAS a rare inherited thrombocytopenia in which splenectomy is still performed. Patients lacking WAS protein have a worse phenotype than those with residual amounts; milder forms are known as X-linked thrombocytopenia. Allogeneic HSC transplantation, first performed for WAS over 40 years ago, has a good prognosis for severe cases with a high risk of bleeding and also benefits immunodeficiency. ${ }^{53}$ In the absence of matched donors gene therapy using a lentiviral vector encoding functional WAS protein is a promising alternative, even if full normalization of the platelet count is not achieved. ${ }^{54}$

\section{Familial platelet disorders with predisposition to myeloid or lymphoid malignancies}

Defects of transcription factors are a major source of inherited thrombocytopenias (Figure 1). Those that inter- fere with the early stages of megakaryocyte maturation are often accompanied by a risk of developing myeloid or lymphoid malignancies and this was first shown for $\mathrm{AD}$ thrombocytopenia caused by mutations of RUNX1. .5.57 $^{5.5}$ The thrombocytopenia is mild and platelet volume increases are modest, but platelet function is defective and a reduced dense granule secretion contributes to the mostly mild bleeding. Disease-causing variants occur across the gene with emphasis on the Runt homology domain that mediates DNA-binding and dimerization with the core binding factor (CBFA $\beta$ ), a complex essential for regulated proliferation and differentiation of HSC and normal megakaryopoiesis as well as suppression of erythroid gene expression. RUNX1 mutations interfere with ploidy and prolong the expression of the cytoskeletal protein MYH10, whose natural loss triggers the switch from mitosis to endomitosis; the result is an abundance of immature megakaryocytes that fail to differentiate properly. ${ }^{21}$ Furthermore, RUNX1 directly or indirectly regulates the synthesis of a number of platelet proteins thereby altering the function of those platelets that are affected. Decreased DNA repair, a pro-inflammatory environment and the prolonged life of HSC predispose patients to acute myeloid leukemia and related conditions. Most of the genetic variants act through haploinsufficiency but, significantly, those that act in a dominant negative manner have a higher probability of causing malignancy. ${ }^{57}$

Myeloid malignancies and myelodysplastic syndromes are also a risk for patients with mutations in the 5' untranslated region of $A N K R D 26$, which are associated with mostly moderate thrombocytopenia, mild bleeding and normal sized platelets (a condition also known as thrombocytopenia 2, THC2). ${ }^{55-60}$ Unexplained findings in some patients are a low density of $\alpha 2 \beta 1$ integrin, decreased numbers of platelet $\alpha$-granules and large, particulate structures formed of ubiquitinated proteins or proteasomes. As for mutations of RUNX1, patients with ANKRD26 mutations have downregulated megakaryocyte maturation leading to an abundance of cells with hypolobulated nuclei. Mechanistically, mutations of the 5 untranslated region of ANKRD26 prevent downregulation of this gene by RUNX1 and FLI1, leading to increased ANKRD26 protein with a lack of repression in the terminal stages of megakaryopoiesis. ${ }^{60}$ Extramedullary hematopoiesis, a characteristic of inherited hemolytic anemia, and myeloproliferative and myelodysplastic syndromes, was reported in a case with ANKRD26-related thrombocytopenia. ${ }^{61}$ It should be noted that THC2 also includes the Ser/Thr kinase MASTL without a tendency for malignancy, dealt with in a later section.

Mutations of ETV6 lead to AD thrombocytopenia and a predisposition to acute lymphoblastic leukemia. ${ }^{2}$ ETV6 is a tumor repressor gene with close links with FLI1. Red cell macrocytosis is said to accompany the thrombocytopenia in some families. An early study involving 130 families with suspected inherited thrombocytopenias identified ETV6 gene variants in seven of them; four of the family members had developed B-cell acute lymphoblastic leukemia during childhood. ${ }^{63}$ Thrombocytopenia was moderate as was the bleeding tendency; platelet size was normal but platelet spreading on fibrinogen was defective. Megakaryocytes in culture produced fewer proplatelets. Mutations within the consensus ETS DNA binding sites of ETV6 resulted in reduced nuclear localization and transcriptional repression with cytoskeletal genes especially 
affected. One mutation (p.R369W) involved a residue previously described as a somatic mutation in different forms of leukemia and cancer. ${ }^{64}$

\section{Linkage with skeletal disorders}

Genetic defects responsible for inherited thrombocytopenias can be syndromic and involve a wide range of other organs and tissues as we have already seen for MYH9-related disease and WAS. Here we provide four examples in which the disease includes well-defined skeletal defects. The Paris-Trousseau syndrome combines macrothrombocytopenia and a panoply of developmental defects including dysmorphogenesis of the face and digits, possible pancytopenia, cardiac abnormalities and mental retardation, which are all characteristic of Jacobsen syndrome. ${ }^{21}$ The inheritance is largely $\mathrm{AD}$ with single allele expression of a small deletion in chromosome 11q23 that includes FLI1, an ETS transcription factor family member and a main regulator of megakaryopoiesis. ${ }^{65}$ Genes targeted by FLI1 include MPL, GPIBA, and ITGA2 and a lack of transactivation early in megakaryocyte development due to a transient hemizygous loss of the affected allele is responsible for the pathology ${ }^{65}$ Dual populations of normal and immature megakaryocytes are generated, the latter with reduced polyploidization and persistence of the cytoskeletal protein, MYH10 (also see the section on RUNX1). Some of the $\alpha$ granules in the enlarged platelets appear fused and giant.

In the thrombocytopenia with absent radius (TAR) syndrome, bilateral radius aplasia and various other developmental defects are accompanied at birth by severe thrombocytopenia but normal sized platelets. Initial studies identified a chromosome 1.21.1 heterozygous microdeletion in patients with TAR syndrome, but the genetics was only fully resolved when WES showed the necessity of a combination of the microdeletion including the RBM8A gene on one allele with one of two rare single nucleotide polymorphisms in regulatory regions of RBM8A on the second allele. ${ }^{66}$ In fact, $R B M 8 A$ codes for $Y 14$, a nuclear and cytoplasmic protein that interacts with mRNA produced by splicing; how it relates to the TAR phenotype remains largely unknown. Hematopoietic progenitors have a reduced response to thrombopoietin and fail to differentiate into megakaryocytes in the marrow. Other hematologic defects include leukocytosis in some patients; enigmatically, the platelet count may correct with age. ${ }^{6}$ TAR syndrome is to be distinguished from radio-ulnar synostosis with amegakaryocytic thrombocytopenia caused by a heterozygous truncating HOXA11 mutation (RUSAT1) or, as determined by WES screening, by de-novo missense mutations in $M E C O M$ (RUSAT2) encoding oncoprotein EVI1 leading to altered activator protein- 1 and transforming growth factor- $\beta$-mediated transcriptional responses. ${ }^{68,99}$ Both HOXA11 and $M E C O M$ mutations cause defects in the early phase of megakaryopoiesis and the risk of trilineage marrow aplasia makes the patients candidates for HSC transplantation.

\section{Defects of platelet biogenesis due to cytoskeletal protein defects}

We have already highlighted how mutations affecting the myosin IIA heavy chain are a frequent cause of macrothrombocytopenia in MYH9-related disease. This prompted research for mutations of genes encoding other cytoskeletal proteins in patients with congenital thrombocytopenia. X-linked mutations in FLNA, encoding filaminA (FLNA), cause a syndromic disorder combined with periventricular nodular heterotopia/otopalatodigital syndromes and other developmental defects. ${ }^{21}$ It can also be associated with Ehlers-Danlos syndrome. We first showed that three patients in France also had macrothrombocytopenia and that bleeding could be severe. ${ }^{70}$ Characterization of their genotype was aided by reports that mice with FlnA-null megakaryocytes prematurely release large and fragile platelets that are rapidly removed from the circulation. ${ }^{11}$ Curiously, platelets of some patients had giant $\alpha$-granules. Cultured megakaryocytes from patients extended proplatelets with large swellings and released giant platelets. ${ }^{71}$ Filamin A promotes branching of actin filaments as well as anchoring membrane receptors and FLNA mutations associate with a loss of platelet adhesive function. Interestingly, a rare gain-of-function missense mutation caused isolated macrothrombocytopenia. ${ }^{71}$ Initially, FLNA mutations were speculated to cause macrothrombocytopenia through loss of the GPIb-FLNA interaction and involvement of RhoA (a GTPase); more recently an alternative mechanism has been proposed based on the loss of $\alpha$ IIb $\beta 3$-filamin A signaling. ${ }^{1,72}$

Hearing loss and macrothrombocytopenia with moderate bleeding were reported in patients from two unrelated families with a heterozygous gain-of-function truncating mutation in DIAPH1; a variant identified by WES followed by crosschecking variants against a database of genes causing deafness. ${ }^{73}$ DIAPH1 is a member of the formin family of cytoskeletal proteins and is a regulator of actin filament formation. The above gain-of-function mutation resulted in cytoskeletal dysregulation and megakaryocytes with reduced but premature proplatelet formation similar to that seen in MYH9-related disease. The defect was linked to a loss of autoinhibition within the diaphanous autoregulatory domain and was distinguished from a frameshift modification that produced hearing loss in a group of families in South America but without hematologic problems. ${ }^{73}$ Brief mention should also be made of moderate macrothrombocytopenia associated with a cluster of missense mutations detected by next-generation sequencing (NGS) in five out of 13 cases with $\mathrm{AD}$ missense mutations in $\mathrm{CDC} 42 .{ }^{74} \mathrm{CDC} 42$ is a small GTPase and a Rho family member and the mutations were said to affect the change between the active and inactive states of the molecule. The disease is syndromic and is associated with various developmental defects including intellectual disability, brain defects, muscle tone abnormalities, facial dysmorphism, and cardiac abnormalities although these were somewhat less apparent for patients with mutations causing macrothrombocytopenia. So far, few platelet function studies appear to have been performed but, on the basis of mouse models, both megakaryocyte maturation and platelet function are likely to be affected. ${ }^{11}$ So far, bleeding has not been reported as a problem for the patients.

Mutations of TUBB1 encoding $\beta 1$-tubulin cause macrothrombocytopenia through altered association with $\alpha$-tubulin and defective microtubule assembly. ${ }^{75,76}$ The defect leads to altered megakaryocyte maturation with the production of fewer proplatelets with large tips but there is a high degree of phenotypic and genotypic variability and while the causal effect of some variants is clear, 
other predicted pathological variants have little effect on platelet count or size. Interestingly, TUBB1 dysfunction may lead to genome instability; co-segregation with myeloid malignancy has been reported, but this tendency needs confirmation as TUBB1 mutations are relatively common. Thyroid dysgenesis has also been linked to TUBB1 mutations in some patients. Variants identified by WES in exons 5 and 6 of $A C T B$ encoding $\beta$-cytoplasmic actin cause syndromic thrombocytopenia by compromising microtubule organization during the final stages of megakaryocyte maturation, but bleeding has not been reported. ${ }^{77}$ The defect has been located in five families and is accompanied by platelet size variability including large forms; associated and highly variable clinical features are given in Online Supplementary Table S1. The defect intervenes in the final stages of platelet biogenesis and preplatelet (a purported intermediate platelet precursor) fragmentation but no defects were seen in megakaryocyte proplatelet formation in culture. ${ }^{77}$ Interestingly, variants in exons 2 to 4 give rise to a severe developmental disorder, Baraitser-Winter cerebrofrontofacial syndrome, but no thrombocytopenia. ${ }^{7}$

Single allele missense mutations in ACTN1 encoding $\alpha$ actinin were first identified in Japanese patients with moderate thrombocytopenia and in a large French pedigree by WES and by genome-wide linkage analysis. ${ }^{78,79}$ Their presence was later revealed as quite common in a non-syndromic form of thrombocytopenia with mild or no bleeding although blood loss can be a problem after surgery. $\alpha$-Actinin is a dimeric protein that cross-links actin filaments into bundles; mutations affecting functional domains act in a dominant-negative manner to disrupt actin filament organization and proplatelet structure in megakaryocytes. Platelet size changes are variable between patients. It is a quite common form of mild inherited thrombocytopenia. ${ }^{78-80}$

A final cytoskeletal gene identified by WES in two human pedigrees with macrothrombocytopenia is TPM4 encoding tropomyosin 4 , another actin-binding protein. ${ }^{81}$ A premature stop codon on a single allele segregated with platelet size increases and a low platelet count. Gene identification was helped by phenotype comparison with a mouse conditional knockout model and confirmed by short hairpin RNA knockdown of TPM4 in normal human megakaryocytes. Platelet function was affected although bleeding was mild. Megakaryocytes in culture produced abundant proplatelets but with fewer branches and enlarged tips, the latter being a standard finding in patients with defects of genes encoding for cytoskeletal proteins, which results in a dynamic re-organization of the cytoskeleton.

Quite remarkably, all of the eight genes encoding cytoskeletal proteins and which give rise to macrothrombocytopenia do so with AD inheritance (Online Supplementary Table S1).

\section{Abnormal integrin interactions with extracellular matrix proteins}

The final steps of megakaryocyte maturation and the migration of these cells towards the vascular sinus depend not only on cytoskeletal proteins, but also on interactions between surface receptors and extracellular matrix con- stituents. Glanzmann thrombasthenia is the classic disorder of platelet function; with AR inheritance; bleeding occurs because thrombus formation and platelet aggregation fail in the absence or non-functioning of integrin $\alpha I I b \beta 3$. Caused by AR mutations in ITGA2B and ITGB3, the platelet count is normal in the absence of major bleeding. ${ }^{82}$ However, rare gain-of-function mutations affecting the structure of $\alpha I I b$ or $\beta 3$ can give rise to macrothrombocytopenia. Our group made the initial report in 1998, describing a single allele p.R995O mutation in the $\alpha \mathrm{IIb}$ cytoplasmic domain; this was later shown to be associated with a null allele. ${ }^{83}$ Platelet aggregation was much decreased with surface $\alpha \operatorname{Ilb} \beta 3$ at 18\%, the macrothrombocytopenia was modest and bleeding infrequent. The affected residue forms an intracellular salt bridge with D723 of $\beta 3$, itself mutated in a variant of Glanzmann thrombasthenia with macrothrombocytopenia (detailed in Nurden et al..$^{22}$ ). Breaking this bond leads to conformational changes that are transmitted to the extracellular domains with a "partial" activation of $\alpha \operatorname{Ilb} \beta 3$. Since these reports, macrothrombocytopenia with $\mathrm{AD}$ inheritance has been shown for other intracytoplasmic or membrane proximal gain-of-function variants affecting $\alpha \mathrm{IIb} \beta 33^{84,85}$ One hypothesis is that the partially activated integrin on megakaryocytes modifies megakaryocyte contact with extracellular matrix proteins in the marrow. This may promote cytoskeletal re-organization with proplatelets having fewer branches and enlarged tips. Interestingly, a gain-offunction mutation affecting $\alpha \operatorname{IIb} \beta 3$ had a dominant phenotypic effect with respect to a loss-of-function variant. ${ }^{86}$

\section{Next-generation sequencing is expanding the landscape of inherited thrombocytopenias}

Careful phenotyping and the use of NGS have led to the identification of a number of new genes responsible for inherited thrombocytopenia with or without large platelets or accompanying platelet function defects. Some have been discussed in preceding sections, others, confined to a limited number of families or of unusual phenotype and with links to signaling pathways, are presented below. All of the upwards of 40 genes now recognized as causative of inherited thrombocytopenia are included in Online Supplementary Table S1.

\section{Microthrombocytopenia}

The classic disorder with a low platelet count and small platelets is WAS, but microthrombocytopenia and a similar phenotype including eczema, leukocytoclastic vasculitis, eosinophilia and elevated IgA and IgE levels was recently linked in two unrelated patients by WES to AR mutations in $A R P C 1 B$, a gene that encodes a subunit of the human actin-related protein 2/3 complex $(\operatorname{Arp} 2 / 3){ }^{87}$ In fact, Arp $2 / 3$ acts with WAS protein in regulating the branching of actin filaments and plays a central role in cell migration, vesicular trafficking and cytokinesis. Platelets from the patients showed defective spreading. Loss of Arp $2 / 3$ function is syndromic in that it predisposed to inflammatory disease althoug many of the immunodeficiencies characteristic of WAS have so far not been observed. Microthrombocytopenia was also linked by NGS in two consanguineous families to a loss-of-function homozygous AR mutation in $F Y B$, encoding the cytosolic 
adaptor protein ADAP (SLAP-130) for the Fyn tyrosine kinase. $^{88}$ The bleeding syndrome in these families was mild. As discussed by the authors, studies using mouse Adap $^{-/}$models showed that the small platelets had a short survival (also a feature of WAS) and defective $\alpha \mathrm{IIb} \beta 3$ activation, findings confirmed in the patients. Subsequent whole sternum three-dimensional confocal microscopy and intravital two-photon microscopy detected reduced megakaryocyte maturation in Adap ${ }^{--}$mice with signs of ectopic release of platelet-like particles within the bone marrow. ${ }^{89}$ Biallelic mutations in PTPRJ, encoding CD148, a master regulator of Src family kinases and the most abundant membrane-bound tyrosine phosphatase in platelets and megakaryocytes, were next shown to result in nonsyndromic microthrombocytopenia in two siblings with mild bleeding. ${ }^{90}$ Loss of CD148 was accompanied by platelet aggregation defects, particularly in relation to GPVI signaling. The thrombocytopenia was again linked to ectopic platelet release within the bone marrow, a characteristic of WAS.

\section{Src, a universal tyrosine kinase}

A gain-of-function, heterozygous E727K mutation was identified in SRC by WES in nine members of a large family; it was linked to thrombocytopenia, a low content of $\alpha$ granules and reduced platelet function. ${ }^{91}$ The thrombocytopenia was syndromic with myelofibrosis, the disease being accompanied by bone defects including mild facial dysmorphia. The marrow showed trilineage dysplasia with immature megakaryocytes poorly able to form proplatelets. Platelets were variable in size and morphologically abnormal; they responded poorly to collagen with reduced GPVI signaling. The gain-of-function mutation was suggested to lift autoinhibition with spontaneous Src activation and upgraded protein tyrosine phosphorylation. The abnormalities were reproduced in a zebrafish model. The mutation may have a feedback on Mpl function.

\section{Mutations affecting ion gradients}

Stormorken syndrome was described many years ago as a "multifaceted bleeding syndrome" in which an unusual form of macrothrombocytopenia was associated with reduced platelet survival and upgraded prothrombin consumption. $^{92}$ It was linked to a spontaneous expression of phosphatidylserine on platelets. Platelet function and thrombus formation under flow were affected. Much later it resurfaced as a syndromic macrothrombocytopenia with a range of clinical features including mild anemia, asplenia, myopathy with tubular aggregates, miosis, immune dysfunction, ichthyosis and dyslexia. ${ }^{93,94}$ Bleeding is life-long but is mostly mild. Heterozygous gain-of-function AD variants in STIM1 identified by WES are the cause of the disease. STIM1 is a $\mathrm{Ca}^{2+}$-sensor within the endoplasmic reticulum; on $\mathrm{Ca}^{2+}$-depletion conformational changes induce its coiled-coil 1 domain to elongate and interact with ORAI1, a surface membrane channel that mediates store-operated calcium entry into cells. ORAI1 can be affected in rare cases with miosis and non-progressive myopathy but without significant platelet abnormalities. The mutations make STIM1 (and secondarily ORAI1) constitutively active, favoring $\mathrm{Ca}^{2+}$ entry into platelets and presumably megakaryocytes. The York platelet syndrome, with large and morphologically abnormal platelets with giant opaque organelles also had gain-of-function mutations in STIM1 thereby expanding the spectrum of
Stormorken syndrome. ${ }^{94}$ WES and comparison with mouse models led us to identify variants in the TRPM7 gene in two French index cases with macrothrombocytopenia and with a possible link to atrial fibrillation in one family. ${ }^{95}$ TRPM7 is both a kinase and an ion channel primarily linked to $\mathrm{Mg}^{2+}$ homeostasis; its absence in mice or diseasecausing variants in man were both accompanied by cytoskeletal changes in megakaryocytes.

\section{Other miscellaneous defects}

Patients in three families with an often strong bleeding history, moderate thrombocytopenia and enlarged platelets were linked by WES to heterozygous mutations within SLFN14 (Schlafen family member 14$).{ }^{96}$ SLFN14 locates to the nucleus, where it acts as an endoribonuclease and is possibly involved in RNA surveillance. The variants in patients with macrothrombocytopenia localize to the ATPase-AAA-4 domain. The protein is known to associate with ribosomes where it regulates RNA degradation; SLFN14 expression was reduced in the patients and a dominant-negative effect was suggested. ${ }^{97}$ Platelet dense granule content was low and platelet aggregation particularly reduced with collagen, modestly reduced and reversible with ADP but normal with arachidonic acid. In a separate study, severe macrothrombocytopenia but with AR inheritance was linked through WES to a homozygous missense mutation in the PRKACG gene encoding the gamma-catalytic subunit of the cyclic adenosine monophosphate (cAMP)-dependent protein kinase A. ${ }^{98}$ Protein kinase A phosphorylates multiple substrates in platelets and in its absence platelet activation and cytoskeletal organization (with extensive degradation of filamin A) were impaired. However the very low platelet count $\left(<10 \times 10^{9} / \mathrm{L}\right)$ precluded platelet aggregation testing. Significantly, megakaryocytes from this patient had a defect in proplatelet formation. Next in this section are AR mutations in $G 6 B(M P I G 6 B)$ encoding the megakaryocyte and platelet inhibitory receptor, G6b-B, a critical regulator of hematopoietic lineage differentiation and megakaryocyte function and platelet production..$^{9}$ The mutations were detected by WES screening of four families with macrothrombocytopenia. One feature was the presence of focal myelofibrosis atypically even in children. Bleeding was moderate and accompanied by anemia and leukocytosis. In a mouse model megakaryocytes were abnormal with reduced proplatelet formation. G6b-B regulates the activity of the tyrosine phosphatases Shp1 and Shp2. Nonetheless, platelet aggregation to ADP was only minimally affected.

Severe thrombocytopenia observed in four Spanish families was associated with a disorder of ceramide biosynthesis with WES linking the disease to AR mutations in KDSR encoding 3-ketodihydrosphingosine reductase, a lipid enzyme that localizes to the endoplasmic reticulum. ${ }^{100}$ Interestingly, in at least two of these cases the platelet count was normal at birth but fell rapidly to around $30 \times 10^{9} / \mathrm{L}$ or lower. Impaired platelet biogenesis was speculated to be due to decreased synthesis of sphingosine-1 phosphate but formal proof of this is lacking. Another disorder in which the platelet count falls after birth is Roifman syndrome in which moderate thrombocytopenia accompanies a variable syndromic disorder characterized by growth retardation, spondylo-epiphyseal dysplasia, cognitive delay and hypogammaglobulinemia. ${ }^{101}$ Interestingly, the disease is driven by AR variants in the small RNA nuclear gene $R N U 4 A T A C$ that intervenes in intron splicing. The platelets 
were unusually round and had altered tubulin and actin levels with DIAPH1 being one of the affected genes.

Finally, mention must be made of IKZF5 encoding a transcription factor known as Pegasus that was highlighted by whole genome sequencing of over 13,000 individuals enrolled in the National Institute for Health Research Bioresource and including 233 cases of isolated thrombocytopenia. ${ }^{102}$ Various causal missense variants were detected in patients with isolated thrombocytopenia but mild or no bleeding; the mutations (sometimes de novo) were proposed to interfere with DNA binding and to selectively alter the expression of a series of proteins expressed in megakaryocytes and platelets. The thrombocytopenia was mild and platelet size was normal. The platelet $\alpha$-granule content was low but the platelet aggregation response was reported as inconsistent. The megakaryocytes produced fewer proplatelets.

\section{Thrombocytopenia, apoptosis and deficient glycosylation}

Early apoptosis with phagocytosis of platelets by macrophages in the spleen or glycosylation changes followed by platelet clearance in the liver are potential causes of inherited thrombocytopenia. Although evidence for such mechanisms remains incomplete, the identification of some mutations, largely by NGS, has revived interest, particularly in deficient glycosylation.

\section{Apoptosis}

Non-syndromic mild AD thrombocytopenia but with little or no bleeding and platelets of normal size is associated with mutations in the CYCS gene encoding mitochondrial cytochrome c, a disease first reported in New Zealand. ${ }^{103,104}$ Although normal-looking megakaryocytes were present in the bone marrow, the number of naked nuclei was increased. Their presence as well as platelets in the marrow space was indicative of ectopic release. Electron microscopy revealed platelets lacking glycogen stores and the microtubule coil. Culture of megakaryocytes from the patients showed that abnormal and normal platelets were both released. Whether or not CYCS mutations, so far limited to three families, lead to loss of mitochondrial inner membrane potential and platelet phosphatidylserine expression remains speculative. Mention must also be made of a missense p.E167D mutation in MASTL, coding for a microtubule-associated Ser/Thr kinase, in a large family first reported many years previously. The mutation was linked to moderate thrombocytopenia (with normal sized platelets), incomplete differentiation of high ploidy megakaryocytes and a propensity to mild bleeding. ${ }^{58,105}$ Recent studies on a mouse knock-in model suggest that this is a gain-of-function mutation leading to platelet survival changes and to platelets that extend unusually long pseudopods and contain hyper-stabilized microtubules. ${ }^{106}$ In mice, Mastl inhibits protein phosphatase 2, an important negative regulator of several major platelet activation pathways. The result is an increased propensity of platelets to form aggregates, reduced platelet survival and increased signs of apoptosis.

\section{Desialylation and other glycosylation changes}

An early study showed macrothrombocytopenia and neutropenia in a patient with a specific defect in $\alpha-2,3$-sialylation and rapid platelet clearance by the AshwellMorell receptor in the liver but the gene defect was not identified. ${ }^{107}$ More recent studies using WES have linked macrothrombocytopenia with AR mutations of GNE encoding UDP-N-acetylglucosamine 2-epimerase/Nacetylmannosamine kinase, a bi-functional enzyme essential for $\mathrm{N}$-acetylneuraminic acid synthesis (the principle sialic acid of human platelets). ${ }^{108-110}$ Interestingly GNE mutations are also a cause of myopathy and in the above studies macrothrombocytopenia was either syndromic or isolated depending on the report. Severe menorrhagia and bleeding into the corpus luteum in affected women were noteworthy. Platelets were large and the numbers of reticulated (young) platelets often increased, features suggesting accelerated platelet clearance. Precise quantification of the platelet sialic acid deficiency and a direct measure of platelet survival have not, however, been made.

A necessary step in the synthesis of oligosaccharides is the transport of cytidine monophosphate-bound sialic acid to the Golgi apparatus. This requires a transporter protein SLC35A1, whose molecular deficiency was recently reported in two siblings with moderate thrombocytopenia, giant platelets, and mild bleeding. ${ }^{111}$ The macrothrombocytopenia is syndromic, featuring delayed psychomotor development, epilepsy, ataxia, microcephaly and choreiform movements. Exome sequencing highlighted a homozygous missense mutation in SLC35A1 in both siblings; their consanguineous parents were heterozygous. Lectin binding confirmed abnormally high $\beta$-galactose exposure on platelets from the patients and increased numbers of reticulated platelets. Bone marrow biopsies showed a high percentage of immature megakaryocytes, yet in culture megakaryocytes from the patients showed normal maturation and proplatelet formation. However quantification of platelet survival using a non-obese diabetic/severe combined immunodeficiency mouse model confirmed that the patients' platelets had a very short lifespan. In a separate study, severe thrombocytopenia with enlarged platelets and dysplastic megakaryocytes was the characteristic of six members of a consanguineous family in whom WES and homozygosity mapping revealed a homozygous mutation in GALE, encoding UDP-galactose-4-epimerase, an enzyme responsible for UDP-galactose inter-conversion with UDP-glucose and UDP-N-acetylgalactosamine inter-conversion with UDP$\mathrm{N}$-acetylglucosamine. ${ }^{112}$ Some individuals also showed mild anemia and febrile neutropenia while galactosemia is a feature of mutations of this gene. With predicted protein instability, the enzyme retained $40 \%$ of its normal activity. Knockdown of GALE in hematopoietic cells slowed the proliferation of these cells in liquid culture; larger colonies possibly reflected an increased presence of immature cells.

\section{Macrothrombocytopenia and sitosterolemia}

An unusual inherited macrothrombocytopenia with epigenetic or environmental implications is sitosterolemia. In this condition, giant platelets associate with AR mutations in $A B C G 5$ and $A B C G 8$, genes which code for proteins that act as cellular transporters of plant sterols, the latter accumulating at high levels in blood and tissues. ${ }^{113}$ Xanthomas, premature atherosclerosis and hemolytic anemia are other 
features; not all patients develop hematologic abnormalities while macrothrombocytopenia can also be isolated. Studies in mice show knock-on effects with sterol intercalation within the demarcation membrane system of megakaryocytes leading to release of hyper-reactive enlarged platelets with deregulation of multiple signaling pathways including GPIb $\alpha$ shedding. ${ }^{114}$

\section{Diagnosis}

The continuing difficulties in diagnosis of inherited thrombocytopenias should not be underestimated. While classic disorders such as BSS, MYH9-related disease and WAS are now relatively straightforward to identify, the majority are not and in the absence of a well-defined family history of bleeding, new cases can still be confused with immune thrombocytopenic purpura. Diagnostic algorithms with steps based on clinical data and laboratory testing aid the work-up and management of patients. ${ }^{115-}$ ${ }^{118}$ The widespread use of whole blood electronic counters to evaluate platelet count and size has helped, while assessing platelet function, distinguishing between syndromic or non-syndromic forms, identifying associated hematologic or marrow defects are important parts of any strategy. A successful diagnosis avoids unnecessary splenectomy and/or the use of drugs given for immune thrombocytopenic purpura. It helps to predict disease evolution, and is indispensable for selecting patients for HSC transplantation or gene therapy. Noteworthy advances have been the application of Human Phenotype Ontology terms and the identification of phenotype clusters of associated pathologies as an aid to establishing phenotype/genotype relationships. ${ }^{119}$ Nonetheless preparing an extensive Human Phenotype Ontology listing is not always compatible with day-to-day hospital practice. Classic tests for evaluating platelet function, such as aggregometry (Born platelet aggregometer, impedance aggregometry or other methods), secretion assays, flow cytometry, western blotting and electron microscopy, are time consuming, expensive to perform and require trained personnel. Critically, many such tests are difficult or impossible to perform when the platelet count is low. Cytochemical or immunofluorescence staining of blood smears is a recognized first step for detecting enlarged platelets, and is particularly useful in third-world countries. $^{32,117}$ The evaluation of platelet spreading on single protein substrates provides more information. ${ }^{120}$ The use of computer-based high-throughput testing of platelet function and thrombus formation under flow on extracellular matrix proteins on microchips is full of promise but has been little tested for thrombocytopenias. ${ }^{121}$

As highlighted throughout our review, applying NGS to inherited thrombocytopenias is greatly expanding the list of causative genes as well as increasing the number of variants implicated in the classic diseases. The initial success of WES in genotyping gray platelet syndrome and the TAR syndromes led to the BRIDGE-Bleeding and Platelet Disorders (BRIDGE-BPD) project, orchestrated by Professor W. Ouwehand, which combines the unique sequencing and bioinformatics resources of the Sanger Institute in Cambridge (UK). ${ }^{122}$ The Genotyping and Platelet Phenotyping (GAPP) consortium led by Professor S. Watson in Birmingham, UK has been another major player. ${ }^{123}$ As the number of causal genes for all forms of inherited platelet disorders increased, BRIDGE-BPD and GAPP both put together platforms to test patients for potentially pathogenic variants against previously identified target genes concentrating on exomes, untranslated regions and selected intronic regions. ${ }^{124-127}$ Indeed, GAPP designed a gene panel specific for inherited thrombocytopenias. ${ }^{127}$ In contrast, the ThromboGenomics Consortium (Department of Haematology, University of Cambridge, UK) included a limited number of genes causal for other blood and thrombotic disorders; the panel was regularly updated and a large cohort of 2,396 patients screened. ${ }^{125,126}$ The use of NGS and high-throughput procedures for diagnosing platelet disorders including thrombocytopenias has quickly expanded worldwide as is illustrated by reports from Italy, Japan, Spain, France, Holland and Scandinavia as well as North America. $46,60,77,78,80,87,108,113,128,129$

The question now is not whether to apply NGS procedures in the mainstream of diagnosis but when and how. ${ }^{128}$ Certainly, a strong argument can now be made to use them upfront: early identification of a causal mutation in a known gene will avoid much unnecessary biological characterization, as we have stated recently in this journal. ${ }^{130}$ It is certainly necessary if prenatal diagnosis or HSC transplantation is on the agenda. Nonetheless, upwards of 200 potential gene variants can be located for each patient, so prioritizing and filtering the variants is key. ${ }^{131}$ Variant selection includes the exclusion of synonymous variants and those with a minor allele frequency $>0.01$ in the normal population. Therefore, in the absence of a previously validated mutation in a known causal gene for inherited thrombocytopenia much care must be taken. Only for a limited number of cases can linkage in large families be performed. Data evaluation in terms of quality control, depth of coverage of each gene, the conservation of affected nucleotides or amino acids, the choice of in silico pathogenicity prediction software, and the classification of variants according to appropriate genetic guidelines have been nicely reviewed elsewhere. ${ }^{125,131}$

In taking account of these advances, the fundamentals for a systematic approach to the diagnosis and management of inherited thrombocytopenia remain largely those expertly outlined in a recent European Hematology Association consensus report on mild and moderate bleeding disorders, to which the reader is directed. ${ }^{132}$ In the context of immune thrombocytopenia, a systematic approach includes careful primary tier assessment of the basic clinic-pathological information for each new patient prior to next stage investigations and, if needed, referral to a specialist center. The recommendations in this European Hematology Association report are very useful in emergency situations and for prevention or control of spontaneous bleeding, such as epistaxis or gum bleeding and atrisk situations, including dental and surgical procedures or traumatic events when immediate practical decisions must be made.

\section{Treatment}

As with all inherited platelet disorders, spontaneous bleeding includes epistaxis, gum bleeding, easy bruising and petechiae while gastrointestinal hemorrhage occurs in some severe cases; bleeding may be prolonged after cuts, trauma, or surgery, while for women, menorrhagia and childbirth are added risks. ${ }^{133,134}$ For example, in a European 
study of pregnancy, the risk of bleeding was greater when mothers had a severe bleeding history and a platelet count below 50x10\% / . Platelet count appeared more important as a parameter than the genetic cause of the inherited thrombocytopenia. ${ }^{133}$ Clearly, studies support the classic theory that a primary role of platelets is to maintain vascular integrity. ${ }^{135}$ Nevertheless, many subjects with new forms of inherited thrombocytopenias have modest reductions in platelet count and will bleed rarely or not at all in normal life. Bleeding in such patients depends not only on the extent of the fall in platelet count but also on the nature of accompanying functional defects. Furthermore, much evidence has accumulated on the nonhemostatic roles of platelets. ${ }^{136}$ Using mouse models, Goerge et al. observed that thrombocytopenia rapidly leads to bleeding in inflamed skin and brain due to the loss of vascular integrity. ${ }^{137}$ Much needs to be learned on how the genetic defects described in our review influence the non-hemostatic roles of platelets.

Managing bleeding in inherited thrombocytopenia is much the same as in all inherited platelet disorders, with platelet and red blood cell transfusions being the first options. The use of recombinant activated factor VII is especially recommended in BSS in which the absence of a major surface constituent (GPIb-IX-V) makes isoantibody formation likely and platelet transfusion ineffective. ${ }^{138}$ Tranexamic acid or local measures are the most frequent options for mild bleeding and tranexamic acid is counseled prior to surgery or childbirth if the thrombocytopenia is severe, with platelet concentrates on standby. A quantitated bleeding score, such as the International Society of Thrombosis and Haemostasis Bleeding Assessment Tool, is useful for assessing disease severity but will not predict bleeding risk. ${ }^{139-141}$ Curing the disease is still at its debut for inherited thrombocytopenia. Human stem cell and allogeneic bone marrow transplants have been used very successfully in children with WAS in whom the immunodeficiency is accompanied by a major bleeding risk. ${ }^{53}$ It has also been used in congenital amegakaryocytic thrombocytopenia and more recently in gray platelet syndrome, in which a positive effect was noted on myelofibrosis, but each procedure has its complications in terms of donor matching and the choice of conditioning regime. ${ }^{21,48,142}$ Lentivirus-based gene therapy is already a proven therapy in WAS when donor-matching for HSC transplantation is a problem, although restoration of the platelet count remains incomplete. ${ }^{54}$ Clearly the gain versus risk profile for the patient must be evaluated case-by-case and current gene therapy procedures can only be considered when a patient's long-term survival is in question or perhaps when CRISPR-Cas gene editing becomes available. One highly promising, noninvasive approach to raising the platelet count is the use of the thrombopoietin-mimetics, eltrombopag or romiplostim. ${ }^{143}$ Although bleeding is generally mild in $M Y H 9$ related disease, eltrombopag was first used successfully prior to surgery in a case with aggravated thrombocytopenia over 10 years ago. ${ }^{144}$ Thrombopoietin-mimetics have more recently been used for a patient with a DIAPH1 mutation prior to hip arthroplasty and as a "bridge" for a child with WAS and severe thrombocytopenia awaiting HSC transplantation. ${ }^{145,146}$ The goal in such situations is to transiently increase the platelet count to $>50 \times 10^{9}$ platelets/L. Romiplostim has been successful in correcting the platelet count resulting from mutations in
THPO in congenital amegakaryocytic thrombocytopenia in which the patient's own thrombopoietin is absent or non-functional. ${ }^{147}$ Overall, a careful and complete diagnosis is essential for the optimal management of patients, not only for those in need of special care but also to avoid over-reacting for patients with little risk of bleeding.

\section{Perspectives}

It is clear that we have entered a new era in the diagnosis of inherited thrombocytopenias with the arrival of WES, whole genome sequencing and selected highthroughput sequencing platforms. A feature of the reports so far is the large genetic heterogeneity. Crucial for the future will be a better understanding of regulatory DNA elements and untranslated regions, a new science that will include epigenomic profiling. ${ }^{148}$ The challenges of whole genome sequencing are great but the potential to identify possible pathological traits hidden up to now, including difficult copy number variations and sequence variations deep within introns, is vast. The study of RNA sorting during platelet biogenesis and of the modulating influence of miRNA is also important. ${ }^{149}$ Despite current technological advances, a high proportion of new cases of inherited thrombocytopenia remain without diagnosis; this proportion is often estimated to be around $50 \%$ but can be as high as $70 \%$ or more depending on the extent to which patients with easily identified disorders have been prescreened. ${ }^{127} 128,150,151$ Assigning a significance to the variants is key, because in the absence of confirmation with follow-up biological studies, including the use of mouse, zebrafish or Drosophila models or site-directed mutagenesis in megakaryocyte-related cells, the alternative is to use sophisticated bioinformatics; new variants are being variously graded as highly significant and likely pathogenic to those of unknown significance. ${ }^{126,131}$ An early metaanalysis of genome-wide association studies identified 68 common single-nucleotide variants that influenced platelet count or platelet volume; genes already linked to inherited thrombocytopenia, such as THPO, GPIBA, $T U B B 1$ and the pro-survival gene, $B A K$, were included, but the majority involved genes with no known link to megakaryopoiesis. ${ }^{152}$ Evaluating the sum of the effects of large combinations of single nucleotide variants and of novel gene variants of unknown significance has barely started yet and they may be disease-modulating, particularly if including heterozygous variants of known causal genes. ${ }^{153}$ Their identification will require large study groups and high-powered bioinformatics beyond the scope of most individual laboratories.

A major challenge posed by inherited thrombocytopenia is identifying how patients, sometimes within the same family, with an identical genotype can vary so much clinically. In a GAPP study, there were descriptions of patients with excessive bleeding, mild thrombocytopenia and platelet dense granule secretion but with an enrichment of heterozygous FLI1 and RUNX1 hypomorphic mutations that were proposed to modulate phenotype. ${ }^{154}$ On the basis of data from the ThromboGenomics project, we identified a disease-modifying TUBB1 mutation in a patient with classic type I Glanzmann thrombasthenia who, confusingly, also had giant platelets and macrothrombocytopenia. ${ }^{155}$ Such studies must be just the tip of the iceberg but the benefit of screening against large 
gene panels is that some patients thought to have a monogenic condition may in fact be seen to have digenic or oligogenic pathologies. The extent of bleeding or predisposition to leukemia may crucially depend on gene variants that are different from those causing the primary disease. An early example is the ARID $5 B$ risk allele for leukemia in patients with ETV6-linked thrombocytopenias. ${ }^{156}$ A future step for modern technologies is to identify variants and gene modulators able to influence phenotype and bleeding - either by protecting against or by exaggerating the primary disease. This will be key to improving prognosis and prevention.

The use of cost-effective NGS procedures, with the continued updating of gene panels, will require national networks with links to high-performance bioinformatics centers able to best analyze the data, perform variant filtering as well as the quality control of the sequencing procedures. As we have proposed elsewhere, this will be best done for Europe with a consensus ${ }^{132}$ in which the European
Hematology Association can be a leader providing guidelines, as is already the case with its comprehensive roadmap for hematology research. ${ }^{157}$ Early recognition of a germline mutation facilitates appropriate treatment, better monitoring for disease progression, proper donor selection for HSC transplantation (family members must also be tested to ensure that they do not carry the mutation), as well as optimal genetic counseling of the affected patients and their family. Quite clearly for many patients the associated pathologies are clinically more serious than the bleeding risk and for some there is an obvious age-dependence. Improving the quality of life of patients and, at the same time, avoiding their stigmatization requires comprehensive ethical considerations. ${ }^{158}$ It is crucial to decide how to provide delicate genetic information concerning a potential risk for leukemia or cancer, especially when potentially causal variants are found in young children, or how to deal with the coincidental identification of gene defects likely to predict other major illnesses.

\section{References}

1. Balduini CL, Melazzini F. Research at the heart of hematology: thrombocytopenias and platelet function disorders. Haematologica. 2017;102(2):203-205.

2. Lee RE, Young RH, Castleman B. James Homer Wright: a biography of the enigmatic creator of the Wright stain on the occasion of its centennial. Am J Surg Pathol. $2002 \cdot 26(1): 88-96$

3. Harrington WJ, Minnich V, Hollingsworth JW, Moore CV. Demonstration of a thrombocytopenic factor in the blood of patients with thrombocytopenic purpura. J Lab Clin Med. 1951;38(1):1-10.

4. Bernard J, Soulier JP. Sur une nouvelle variété de dystrophie thrombocytaire hémorragipare congénitale. Sem Hop. 1948;24 (Spec. No.):3217-3223.

5. Gröttum KA, Solum NO. Congenital thrombocytopenia with giant platelets: a defect in the platelet membrane. $\mathrm{Br} \mathrm{J}$ Haematol. 1969;16(3):277-290.

6. Nurden AT, Caen JP. Specific roles for platelet surface glycoproteins in platelet function. Nature. 1975;255(5511):720-722.

7. Chang Y, Bluteau D, Debili N, Vainchenker W. From haematopoietic stem cells to platelets. J Thromb Haemost. 2007;5(Suppl 1):318-327

8. Kaushansky K, Lok S, Holly RD, et al. Promotion of megakaryocyte progenitor expansion and differentiation by the c-Mpl ligand thrombopoietin. Nature. 1994;369 (6481):568-571.

9. Machlus KR, Italiano JE. The incredible journey: from megakaryocyte development to platelet formation. J Cell Biol. 2013;201(6):785-796

10. Junt T, Schulze H, Chen Z, et al. Dynamic visualization of thrombopoiesis within bone marrow. Science. 2007;317(5845): 1767-1770.

11. Dütting S, Gaits-Iacovoni F, Stegner D, et al. A Cdc42/RhoA regulatory circuit downstream of glycoprotein $\mathrm{Ib}$ guides transendothelial platelet biogenesis. Nat Commun. 2017;8:15838.
12. Lefrancais E, Ortiz-Munoz G, Caudrillier A, et al. The lung is a site of platelet biogenesis and a reservoir for haematopoietic progenitors. Nat New Biol. 2017;544(7648): 105-109.

13. Nishimura S, Nagasaki M, Kunishima S, et al. IL- $1 \alpha$ induces thrombopoiesis through megakaryocyte rupture in response to acute platelet needs. J Cell Biol. 2015;209(3):453-466.

14. Grozovsky R, Begonja AJ, Liu K, et al. The Ashwell-Morell receptor regulates hepatic thrombopoietin production via JAK2STAT3 signaling. Nat Med. 2015;21(1):4754.

15. Li Y, Fu Y, Ling T, et al. Sialylation on Oglycans protects platelets from clearance by liver Kupffer cells. Proc Natl Acad Sci U S A 2017;114(31):8360-8365.

16. Deppermann C, Kratofil RM, Peiseler M, et al. Macrophage galactose lectin is critical for Kupffer cells to clear aged platelets. J Exp Med. 2020;217(4):e20190723.

17. McArthur K, Chappaz S, Kile BT. Apoptosis in megakaryocytes and platelets: the life and death of a lineage. Blood. 2018;131(6):605-610

18. Mason KD, Carpinelli MR, Fletcher JI, et al. Programmed anuclear cell death delimits platelet life span. Cell. 2007;128(6):11731186.

19. Jackson SP, Schoenwaelder S. Procoagulant platelets: are they necrotic? Blood. 2010:116(12):2011-2018.

20. Berndt MA, Andrews RK. Bernard-Soulier syndrome. Haematologica. 2011;96(3):355359.

21. Favier R, Raslova H. Progress in understanding the diagnosis and molecular genetics of macrothrombocytopenias. $\mathrm{Br} \mathrm{J}$ Haematol. 2015;170(5):626-639.

22. Savoia A, Kunishima S, De Rocco D, et al. Spectrum of mutations in Bernard-Soulier syndrome. Hum Mutat. 2014;35(9):10331045.

23. Poujol C, Ware J, Nieswandt B, Nurden AT, Nurden P. Absence of GPIbo is responsible for aberrant membrane development during megakaryocyte maturation: ultrastructural study using a transgenic model. Exp
Hematol. 2002;30(4):352-360.

24. Sivapalaratnam S, Westbury SK, Stephens JC, et al. Rare variants in GP1BB are responsible for autosomal dominant macrothrombocytopenia. Blood. 2017;129(4):520-524.

25. Othman M. Platelet-type von Willebrand disease: three decades in the life of a rare bleeding disorder. Blood Rev. 2011;25(4) 147-153.

26. Bury L, Malara A, Momi S, Petito E, Balduini A, Gresele P. Mechanisms of thrombocytopenia in platelet-type von Willebrand disease. Haematologica. 2019;104(7):1473-1481

27. Federici A, Mannucci PM, Castaman G, et al. Clinical and molecular predictors of thrombocytopenia and risk of bleeding in patients with von Willebrand disease type 2B : a cohort study of 67 patients. Blood. 2009;113(3):526-534.

28. Nurden P, Gobbi G, Nurden A, et al Abnormal VWF modifies megakaryocytopoiesis: studies of platelets and megakaryocyte cultures from patients with von Willebrand disease type $2 \mathrm{~B}$. Blood. 2010;115(13):2649-2656.

29. Casari C, Berrou E, Lebret M, et al. Von Willebrand factor mutation promotes thrombocytopathy by inhibiting integrin $\alpha \operatorname{Ilb} \beta 3$. J Clin Invest. 2013;123(12):50715081

30. Dupont A, Soukaseum C, Cheptou M, et al. Relevance of platelet desialylation and thrombocytopenia in type $2 \mathrm{~B}$ von Willebrand disease: preclinical and clinical evidence. Haematologica. 2019;104(12): 2493-2500.

31. Pecci A, Ma X, Savoia A, Adelstein RS MYH9: structure, functions and role of non-muscle myosin IIA in human disease. Gene. 2018;664:152-167.

32. Greinacher A, Pecci A, Kunishima S, et al Diagnosis of inherited platelet disorders on a blood smear: a tool to facilitate world wide diagnosis of platelet disorders. I Thromb Haemost. 2017;15(7):1511-1521.

33. Heath KE, Campos-Barros A, Toren A, et al Non-muscle myosin heavy chain IIA mutations define a spectrum of autosomal dominant macrothrombocytopenias: May- 
Inherited thrombocytopenias

Hegglin anomaly and Fechtner, Sebastien, Epstein and Alport-like syndromes. Am J Hum Genet. 2001;69(5):1033-1045.

34. Saposnik B, Binard S, Fenneteau $O$, et al. French MYH9 network. Mutation spectrum and genotype-phenotype correlations in a large French cohort of MYH9-related disorders. Mol Genet Genomic Med. 2014;2(4):297-312.

35. Aguilar A, Pertuy F, Eckly A, et al Importance of environmental stiffness for megakaryocyte differentiation and proplatelet formation. Blood. 2016;128(16): 2022-2032.

36. Breton-Gorius J, Vainchenker W, Nurden A, Levy-Toledano S, Caen J. Defective alphagranule production in megakaryocytes from gray platelet syndrome: ultrastructural studies of bone marrow cells and megakaryocytes growing in culture from blood cell precursors. Am J Pathol. 1981;102(1):10-19.

37. Gunay-Aygum M, Zivony-Elboum Y, Gumruk F, et al. Gray platelet syndrome: natural history of a large patient cohort and locus assignment to chromosome $3 \mathrm{p}$. Blood. 2010;116(23):4990-5001.

38. Chen CH, Lo RW, Urban D, Pluthero FG, Kahr WH. $\alpha$-granule biogenesis: from disease to discovery. Platelets. 2017;28(2):147154.

39. Deppermann C, Cherpokova D, Nurden P, et al. Gray platelet syndrome and defective thrombo-inflammation in Nbeal2-deficient mice. J Clin Invest. 2013;123(8):3331-3342.

40. Di Buduo CA, Alberelli AC, Glembotsky AC, et al. Abnormal proplatelet formation and emperipolesis in cultured human megakaryocytes from gray platelet syndrome patients. Sci Rep. 2016;6:23213.

41. Sowerby JM, Thomas DC, Clare S, et al. NBEAL2 is required for neutrophil and NK cell function and pathogen defense. J Clin Invest. 2017;127(9):3521-3526.

42. Wijgaerts A, Wittevrongel C, Thys C, et al. The transcription factor GATA1 regulates NBEAL2 expression through a long-distance enhancer. Haematologica. 2017;102 (4):695-706.

43. Nichols KE, Crispino JD, Poncz M, et al. Familial dyserythropoietic anaemia and thrombocytopenia due to an inherited mutation in GATA1. Nat Genet. 2000;24(3):266-270.

44. Stevenson WS, Morel-Kopp MC, Chen O, et al. GFI1B mutation causes a bleeding disorder with abnormal platelet function. J Thromb Haemost. 2013;11(11):2039-2047.

45. Monteferrario D, Bolar NA, Marneth AE, et al. A dominant negative GFI1B mutation in the gray platelet syndrome. New Engl J Med. 2014;370(3):245-253.

46. Van Oorschott R, Marneth AE, Bergevoet $\mathrm{SM}$, et al. Inherited missense variants that affect GFI1B function do not necessarily cause. bleeding diatheses. Haematologica. 2019;104(6):e260-e264.

47. Ballmaier M, Germeshausen M, Schulze H, et al. c-Mpl mutations are the cause of congenital amegakaryocytic thrombocyopenia. Blood. 2001;97(1):139-146.

48. Plo I, Bellané-Chantelot, Mosca M, Mazzi S, Marty C, Vainchenker W. Genetic alterations of the thrombopoietin/MPL/JAK2 axis impacting megakaryopoiesis. Front Endocrinol (Lausanne). 2017;8:234.

49. Imai K, Morio T, Zhu Y, et al. Clinical course of patients with WASP gene mutations. Blood. 2004;103(2):456-464.

50. Thrasher AJ, Burns SO. WASP: a key immunological multitasker. Nat Rev
Immunol. 2010;10(3):182-192.

51. Mahlaoui N, Pellier I, Mignot C, et al. Characteristics and outcome of early-onset, severe forms of Wiskott-Aldrich syndrome. Blood. 2013;121(9):1510-1516.

52. Sabri S, Foudi A, Boukour S, et al. Deficiency in the Wiskott-Aldrich protein induces premature proplatelet formation and platelet production in the bone marrow compartment. Blood. 2006;108(1):134140.

53. Aiuti A, Biasco L, Scarauzza S, et al. Lentiviral hematopoietic stem cell gene therapy in patients with Wiskott-Aldrich syndrome. Science. 2013;341(6148): 1233151.

54. Ferrua F, Cicalese MP, Galimberti S, et al. Lentiviral haemopoietic stem/progenitor cell gene therapy for treatment of WiskottAldrich syndrome: interim results of a nonrandomized, open-label, phase $1 / 2$ clinical study. Lancet Haematol. 2019;6(5):e239e253.

55. Song WJ, Sullivan MG, Legare RD, et al. Haploinsufficiency of CBFA2 causes familial thrombocytopenia with propensity to develop acute myelogenous leukemia. Nat Genet. 1999;23(2):166-175.

56. Lordier L, Bluteau D, Jalil A, et al. RUNX1induced silencing of non-muscle myosin heavy chain IIB contributes to megakaryocyte polyploidization. Nat Commun. 2012;3:717.

57. Morgan NV, Daly M. Gene of the issue: RUNX1 mutations and inherited bleeding. Platelets. 2017;28(2):208-210.

58. Drachman JG, Jarvik GP, Mehaffey MG. Autosomal dominant thrombocytopenia: incomplete megakaryocyte differentiation and linkage to chromosome 10. Blood. 2000;96(1):118-125.

59. Noris P, Perrotta S, Seri M, et al. Mutations in ANKRD26 are responsible for a frequent form of inherited thrombocytopenia: analysis of 78 patients from 21 families. Blood. 2011;117(24):6673-6680.

60. Bluteau D, Balduini A, Balayn A, et al. Thrombocytopenia-associated mutations in the ANKRD26 regulatory region induce MAPK hyperactivation. J Clin Invest. 2014;124(2):580-591.

61. Zaninetti C, Melazzini F, Croci GA, Boveri E, Balduini CL. Extramedullary hematopoiesis: a new feature of inherited thrombocytopenias. J Thromb Haemost. 2017;15(11):2226-2229.

62. Di Paola J, Porter CC. ETV6-related thrombocytopenia and leukemia predisposition. Blood. 2019;134(8):663-667.

63. Noetzli L, Lo RW, Lee-Sherick AB, et al. Germline mutations in ETV6 are associated with thrombocytopenia, red cell macrocytosis and predisposition to lymphoblastic leukemia. Nat Genet. 2015;47(5):535-538.

64. Melazzini F, Palombo F, Balduini A, et al. Clinical and pathogenic features of ETV6related thrombocytopenia with predisposition to acute lymphoblastic leukemia. Haematologica. 2016;101(11):1333-1342.

65. Raslova H, Komura E, Le Couedic JP, et al. Fli1 monoallelic expression combined with its hemizygous loss underlies ParisTrousseau/Jacobsen thrombopenia. J Clin Invest. 2004;114(1):77-84.

66. Albers CA, Paul DS, Schulze $\mathrm{H}$, et al. Compound inheritance of a low-frequency regulatory SNP and a rare null mutation in exon-junction complex subunit RBM8A causes TAR syndrome. Nat Genet. 2012;44(4):435-439.

67. Manukjan G, Bösing $H$, Schmugge $M$,

Straub G, Schulze H. Impact of genetic variants on haematopoiesis in patients with thrombocytopenia absent radii (TAR) syndrome. Br J Haematol. 2017;179(4):606617.

68. Thompson AA, Nguyen LT. Amegakaryocytopenia and radio-ulnar synostosis are associated with HOXA11 mutation. Nat Genet. 2000;26(4):397-398.

69. Nijhori T, Ouchi-Uchiyama M, Sasahara Y, et al. Mutations in MECOM, encoding oncoprotein EVI1, cause radioulnar synostosis with amegakaryocytic thrombocytopenia. Am J Hum Genet. 2015;97(6):848854.

70. Nurden P, Debili N, Coupry I, et al. Thrombocytopenia resulting from mutations in filamin A can be expressed as an isolated syndrome. Blood. 2011;118(22): 5928-5937.

71. Begonja AJ, Hoffmeister KM, Hartwig JH, Falet H. FlnA-null megakaryocytes prematurely release large and fragile platelets that circulate poorly. Blood. 2011;118(8):22852295.

72. Donada A, Balayn N, Silwa D, et al. Disrupted filamin $A / \alpha \operatorname{Ilb} \beta 3$ interaction induces macrothrombocytopenia by increasing RhoA activity. Blood. 2019;133 (16):1778-1788.

73. Stritt $S$, Nurden P, Turro E, et al. A gain-offunction variant in DIAPH1 causes dominant macrothrombocytopenia and hearing loss. Blood. 2016;127(23):2903-2914.

74. Martinelli S, Krumbach OHF, Pantaleoni F, et al. Functional dysregulation of CDC42 causes diverse developmental phenotypes. Am J Hum Genet. 2018;102(2):309-320.

75. Kunishima S, Kobayashi R, Itoh TJ, Hamaguchi M, Saito H. Mutation of the beta1-tubulin gene associated with congenital macrothrombocytopenia affecting microtubule assembly. Blood. 2009;113 (2):458-461.

76. Burley K, Westbury SK, Mumford AD. TUBB1 variants and human platelet traits. Platelets. 2018;29(2):209-211.

77. Latham SL, Ehmke M, Reinke PYA, et al. Variants in exons 5 and 6 of ACTB cause syndromic thrombocytopenia. Nat Commun. 2018;9(1):4250.

78. Kunishima S, Okuno Y, Yoshida K, et al. ACTN1 mutations cause congenital macrothrombocytopenia. Am J Hum Genet. 2013;92(3):431-438.

79. Guéguen P, Roualt K, Chen JM, et al. A missense mutation in the alpha-actinin 1 gene (ACTN1) is the cause of autosomal dominant macrothrombocytopenia in a large French family. PloS One. 2013;8(9):e74728.

80. Bottega R, Marconi C, Faleschini M, et al. ACTN1-related thrombocytopenia: identification of novel families for phenotypic characterization. Blood. 2015;125(5):869872.

81. Pleines I, Woods J, Chappaz S, et al. Mutations in tropomyosin 4 underlie a rare form of human macrothrombocytopenia. J Clin Invest. 2017;127(3):814-829.

82. Nurden AT, Fiore M, Nurden P, Pillois X. Glanzmann thrombasthenia: a review of ITGA2B and ITGB3 defects with emphasis on variants, phenotypic variability, and mouse models. Blood. 2011;118(23):59966005.

83. Nurden AT, Pillois $X$, Fiore $M$, et al. Expanding the mutation spectrum of the $\alpha I I b \beta 3$ integrin in Glanzmann thrombasthenia: screening of the ITGA2B and ITGB3 genes in a large international cohort. Hum Mutat. 2015;36(5):548-561. 
84. Nurden P, Bordet JC, Pillois X, Nurden AT. An intracytoplasmic $\beta 3$ Leu718 deletion in a patient with a novel platelet phenotype. Blood Adv. 2017;1(8):494-499.

85. Bury L, Falcinelli E, Chiasserini D, Springer TA, Italiano JE Jr, Gresele P. Cytoskeletal perturbation leads to platelet dysfunction and thrombocytopenia in variant forms of Glanzmann thrombasthenia. Haematologica. 2016;101(1):46-56.

86. Bury L, Zetterburg E, Leinoe EB, et al. A novel variant Glanzmann thrombasthenia due to co-inheritance of a loss and a gainof-function mutation of ITGB3: evidence of a dominant effect of gain-of-function mutations. Haematologica. 2018;103(6):e259e263.

87. Kahr WH, Pluthero FG, Elkadri A, et al. Loss of the Arp2/3 complex component ARPC1B causes platelet abnormalities and predisposes to inflammatory disease. Nat Commun. 2017;8:14816.

88. Hamamy H, Makrythanasis P, Al-Allawi N, Muhsin AA, Antonarakis SE. Recessive thrombocytopenia likely due to a homozygous pathogenic variant in the FYB gene: case report. BMC Med Genet. 2014;15:135.

89. Spindler M, van Eeuwijk JMM, Schurr Y, et al. ADAP deficiency impairs megakaryocyte polarization with ectopic proplatelet release and causes microthrombocytopenia. Blood. 2018;132(6):635-646.

90. Marconi C, Di Buduo CA, LeVine K, et al. Loss-of-function mutations in PTPRJ cause a new form of inherited thrombocytopenia. Blood. 2019;133(12):1346-1357.

91. Turro E, Greene D, Wijgaerts A, et al. A dominant gain-of-function in universal tyrosine kinase SRC causes thrombocytopenia, myelofibrosis, bleeding and bone pathologies. Sci Transl Med. 2016;8 (328):328ra330.

92. Stormorken H, Holmsen H, Sund R, et al. Studies on the haemostatic defect in a complicated syndrome. An inverse Scott syndrome platelet membrane abnormality. Thromb Haemost. 1995;74(5):1244-1251.

93. Nesin V, Wiley G, Kousi M, Ong EC, et al. Activating mutations in STIM1 and ORAI1 cause overlapping syndromes of tubular myopathy and congenital miosis. Proc Natl Acad Sci U S A. 2014:111(11):4197-4202.

94. Markello T, Chen D, Kwan JW et al. York platelet syndrome is a CRAC channelopathy due to gain-of-function mutations in STIM1. Mol Genet Metab. 2015;114(3): 474-482.

95. Stritt S, Nurden P, Favier R, et al. TRPM7 channel function deregulates thrombopoiesis through altered cellular homeostasis and cytoskeletal architecture. Nat Commun. 2016;7:11097.

96. Fletcher SJ, Johnson B, Lowe GC, et al. SLFN14 mutations underlie thrombocytopenia with excessive bleeding and platelet secretion defects. J Clin Invest. 2015;125(9):3600-3605.

97. Fletcher SJ, Pisareva VP, Khan AQ, Tcherepanov A, Morgan NV, Pisarev AV. Role of the novel endoribonuclease SLN14 and its disease-causing mutations in ribosomal degradation. RNA. 2018;24(7):939-949.

98. Manchev VT, Hilpert M, Berrou E, et al. A new form of macrothrombocytopenia induced by a germline mutation in the PRKACG gene. Blood. 2014;124(16):25542563.

99. Hofmann I, Geer MJ, Vögtle T, et al. Congenital macrothrombocytopenia with focal myelofibrosis due to mutations in human G6b-B is rescued in humanized mice. Blood. 2018:132(13):1399-1412.

100. Takeichi T, Torrelo A, Lee JYW et al. Biallelic mutations in KDSR disrupt ceramide synthesis and result in a spectrum of keratinization disorders associated with thrombocytopenia. J Invest Dermatol. 2017;137(11):2344-2353

101. Heremans J, Garcia-Perez JE, Turro E, et al. Abnormal differentiation of $\mathrm{B}$ cells and megakaryocytes in patients with Roifman syndrome. J Allergy Clin Immunol. 2018;142(2):630-646.

102.Lentaigne C, Greene D, Sivapalaratnam S, et al. Germline mutations in the transcription factor IKZF5 cause thrombocytopenia. Blood. 2019:134(23):2070-2081.

103. Morison IM, Cramer-Bordé EM, Cheesman EJ, et al. A mutation of human cytochrome C enhances the intrinsic apoptotic pathway but causes only thrombocytopenia. Nat Genet. 2008;40(4);387-389.

104.Ledgerwood EC, Dunstan-Harrison C, Ong L, Morison IM. CYCS gene variants associated with thrombocytopenia. Platelets. 2019;30(5):672-674.

105. Gandhi MJ, Cummings CL, Drachman JG. FLJ14813 missense mutation: a candidate for autosomal dominant thrombocytopenia on chromosome 10. Hum Hered. 2003;55 (1):66-70.

106. Hurtado B, Trakala M, Ximenez-Embun P, et al. Thrombocytopenia-associated mutations in Ser/Thr kinase MASTL deregulate actin cytoskeletal dynamics in platelets. J Clin Invest. 2018;128(12):5351-5367.

107. Jones C, Denecke J, Strater R, et al. A novel type of macrothrombocytopenia associated with a defect in alpha2,3 sialylation. Am J Pathol. 2011;179(4):1969-1977.

108. Izumi R, Niihori T, Suzuki N, et al. GNE myopathy associated with congenital thrombocytopenia: a report of two sublings. Neuromuscul Disord. 2014;24(12): 1068-1072

109. Revel-Vilk S, Shai E, Turro E, et al. GNE variants causing autosomal recessive macrothrombocytopenia without associated muscle wasting. Blood. 2018;132(17): 1851-1854.

110. Futterer J, Dalby A, Lowe GC, et al. UK GAPP Study group. Mutation in GNE is associated with severe congenital thrombocytopenia. Blood. 2018;132(17):1855-1858.

111. Kauskot A, Pascreau T, Adam F, et al. A mutation in the gene coding for the sialic acid transporter SLC35A1 is required for platelet survival but not proplatelet formation. Haematologica. 2018;103(12):e613 e617.

112. Seo A, Gulsuner S, Pierce S, et al. Inherited thrombocytopenia associated with mutation of UDP-galactose-4-epimerase (GALE). Hum Molec Genet. 2019;28(1):133-142.

113. Bastida JM, Benito R, Janusz K, et al Two novel variants of the ABCG5 gene cause xanthelasmas and macrothrombocytopenia: a brief review of hematologic abnormalities of sitosterolemia. J Thromb Haemost. 2017;15(9):1859-1866.

114. Kanaji T, Kanaji S, Montgomery RR, Patel SB, Newman PJ. Platelet hyperreactivity explains the bleeding abnormality and macrothrombocytopenia in a murine model of sitosterolemia. Blood. 2013;122 (15):2732-2742

115. Balduini CL, Cattaneo M, Fabris F, et al Inherited thrombocytopenias: a proposed diagnostic algorithm from the Italian Gruppo di Studio delle Plastrine. Haematologica. 2003;88(5):582-592.

116. Bolton-Maggs PHB, Chalmers EA, Collins
PW, et al. A review of inherited platelet disorders with guidelines for their management on behalf of the UKHCDO. Br J Haematol. 2006;135(5):603-633.

117. Noris P, Biino G, Pecci A, et al. Platelet diameters in inherited thrombocytopenias: analysis of 376 patients with all known disorders. Blood. 2014;124(6):e4-e10.

118. Gresele P. Diagnosis of inherited platelet function disorders: guidance from the SSC of the ISTH. J Thromb Haemost. 2015;13(2):314-322.

119. Westbury SK, Turro E, Greene D, et al Human Phenotype Ontology annotation and cluster analysis to unravel genetic defects in 707 cases wth unexplained bleeding and platelet disorders. Genome Med. 2015;7(1):36.

120. Khan AO, Maclachlan A, Lowe JC, et al. High-throughput platelet spreading analysis: a tool for the diagnosis of platelet-based bleeding disorders. Haematologica. 2020; 105(3):e124-e128.

121. Van Geffen JP, Brouns SLN, Batista J, et al High-throughput elucidation of thrombus formation reveals sources of platelet function variability. Haematologica. 2019;104 (6):1256-1267.

122.Lentaigne C, Freson K, Laffan MA, Turro E Ouwehand WH. On behalf of the BRIDGEBPD Consortium and the ThromboGenomics Consortium. Inherited platelet disorders: towards DNA-based diagnosis. Blood. 2016;127(33):2814-2823.

123.Johnson B, Lowe GC, Futterer J, et al. Whole exome sequencing identifies genetic variants in inherited thrombocytopenia with secondary qualitative function defects. Haematologica. 2016;101(10): 1170-1179.

124. Simeoni I, Stephens JC, Hu F, et al. A highthroughput sequencing test for diagnosing inherited bleeding, thrombotic and platelet disorders. Blood. 2016;127(23):2791-2803.

125. Downes K, Megy K, Duarte D, et al Diagnostic high-throughput sequencing of 2,396 patients with bleeding, thrombotic and platelet disorders. Blood. 2019;134(23): 2082-2091.

126. Megy K, Downes K, Simeoni I, et al. Curated disease-causing genes for bleeding, thrombotic, and platelet disorders: communication from the SSC of the ISTH. Thromb Haemost. 2019;17(8):1253-1260.

127. Johnson B, Doak R, Allsup D, et al. A comprehensive targeted next-generation sequencing panel for genetic diagnosis of patients with suspected inherited thrombocytopenia. Res Pract Thromb Haemost. 2018;2(4):640-652.

128. Bastida JM, Lozano ML, Benito R, et al. Introducing high-throughput sequencing into mainstream genetic diagnosis practice in inherited platelet disorders. Haematologica. 2018;103(1):148-162.

129. Leinoe E, Gabrielate M, Ostrup O, et al Outcome of an enhanced diagnostic pipeline for patients suspected of inherited thrombocytopenia. $\mathrm{Br} \mathrm{J}$ Haematol. 2019;186(2):373-376.

130. Nurden AT, Nurden P. High-throughput sequencing for rapid diagnosis of inherited platelet disorders: a case for a European consensus. Haematologica. 2018;103(1):68.

131. Freson K, Turro E. High-throughput sequencing approaches for diagnosing hereditary bleeding and platelet disorders. Thromb Haemost. 2017;15(7):1262-1272.

132. Rodeghiero F, Pabinger I, Ragni M, et al. Fundamentals for a systematic approach to 
mild and moderate inherited bleeding disorders: an EHA consensus report. HemaSphere. 2019;3(5):e286.

133. Noris P, Schlegel N, Kiersy C, et al. Analysis of 339 pregnancies in 181 women with 13 different forms of inherited thrombocytopenia. Haematologica. 2014;99(8):13871394.

134. Orsini S, Noris P, Bury L, et al. Bleeding risk of surgery and its prevention in patients with inherited platelet disorders. Haematologica. 2017;102(7):1192-1203.

135. Gimbrone MA Jr, Aster RH, Cotran RS, Corkery J, Jandl JH, Folkman J. Preservation of vascular integrity in organs perfused in vitro with a platelet-rich medium. Nature. 1969;222(5188):33-36.

136. Nurden AT. The biology of the platelet with special reference to inflammation, wound healing and immunity. Front Biosci (Landmark Ed). 2018:23:726-751.

137. Goerge T, Ho-Tin-Noe B, Carbo C, et al. Inflammation induces hemorrhage in thrombocytopenia. Blood. 2008;111(10): 4958-4964

138. Poon MC, D'Oiron R. Alloimmunization in congenital deficiencies of platelet surface glycoproteins: focus on Glanzmann's thrombasthenia and Bernard-Soulier's syndrome. Semin Thromb Haemost. 2018;44(6):604-614.

139. Rodeghiero F, Tosetto A, Abshire T, et al. ISTH/SSC joint VWF and Perinatal/ Pediatric Hemostasis Subcommittees Working Group. ISTH/SSC bleeding assessment tool: a standardized questionnaire and a proposal for a new bleeding score for inherited bleeding disorders. J Thromb Haemost. 2010;8(9):2063-2065.

140. Gresele P, Orsini S, Noris P, et al. Validation of the ISTH/SSC bleeding assessment tool for inherited platelet disorders: a communication from the Platelet Physiology SSC. J
Thromb Haemost. 2020;18(3):732-739.

141. Fasulo MR, Biquzzi E, Abbattista M, et al. The ISTH bleeding assessment tool and the risk of future bleeding. J Thromb Haemost. 2018;16(1):125-130

142. Favier R, Roussel X, Audia S, et al. Correction of severe myelofibrosis, impaired platelet function abnormalities in a patient with gray platelet syndrome successfully treated by stem cell transplantation. Platelets. 2019 Sep 10:1-5 [Epub ahead of print].

143. Ghanima W, Cooper N, Rodeghiero F, Godeau B, Bussel JB. Thrombopoietin receptor agonists: ten years later. Haematologica. 2019;104(6):1112-1123.

144.Pecci A, Gresele P, Kiersy C, et al Eltrombopag for the treatment of the inherted thrombocytopenia deriving from MYH9 mutations. Blood. 2010;116(26): 5832-5837.

145. Westbury SK, Downes K, Burney C, et al. Phenotypic description and response to thrombopoietin receptor agonist in DIAPH1-related disorder. Blood Adv. 2018;2(18):2341-2346.

146. Gabelli M, Marzollo A, Notarangelo LD, Basso G, Putti MC. Eltrombopag use in a patient with Wiskott-Aldrich syndrome. Pediatr Blood Cancer. 2017;64(12)

147.Pecci A, Ragab I, Bozzi V, et al. Thrombopoietin mutation in congenital amegakaryocytic thrombocytopenia treatable with romiplostim. EMBO Mol Med. 2018;10(1):63-75

148.Barbosa M, Joshi RS, Garg P, et al. Identification of rare de novo epigenetic variations in congenital disorders. Nat Commun. 2018;9(1):2064

149. Fisher MS, Di Paola J. Genomics and transcriptomics of megakaryocytes and platelets: implications for health and disease. Res Pract Thromb Haemost. 2018;2
(4):630-639

150. Almazni I, Stapley R, Morgan NV. Inherited thrombocytopenia: update on genes and genetic variants which may be associated with bleeding. Front Cardiovasc Med. 2019;6:80.

151. Savoia A. Molecular basis of inherited thrombocytopenias: an update. Curr Opin Hematol. 2016;23(5):486-492

152. Gieger C, Radakrishnan A, Cvejic A, et al. New gene functions in megakaryopoiesis and platelet formation. Nature. 2011;489 (7376):201-208.

153. Astle WJ, Elding H, Jiang T, et al. The allellic landscape of human blood cell trait variation and links to common complex disease. Cell. 2016;167(5):1415-1429

154. Stockley J, Morgan NV, Bem D, et al. Enrichment of FLI1 and RUNX1 mutations in families with excessive bleeding and platelet dense granule secretion defects. Blood. 2013;122(25):4090-4093.

155. Guillet B, Bayart S, Pillois X, Nurden P Caen IP, Nurden AT. A Glanzmann thrombasthenia family associated with a TUBB1 related macrothrombasthenia. J Thromb Haemost. 2019;17(12):2211-2215

156. Karastaneva A, Nebral K, Schlagenhauf A et al. Novel phenotypes observed in patients with ETV6-linked leukemia/familial thrombocytopenia syndrome and a biallelic ARID5B risk allele as leukaemogenic cofactor. I Med Genet. 2019;imedgenet2019-106339. [Epub ahead of print]

157. Engert A, Balduini C, Brand A, et al. The European Haematology Association roadmap for haematology research: a consensus document. Haematologica. 2016 101 (2):115-208.

158. Greinacher A, Eekels JJM. Simplifying the diagnosis of inherited platelet disorders The new tools do not make it easier. Blood. 2019;133(23):2478-2483. 